\title{
APPROXIMATION AND EQUIDISTRIBUTION OF PHASE SHIFTS: SPHERICAL SYMMETRY
}

\author{
KIRIL DATCHEV, JESSE GELL-REDMAN, ANDREW HASSELL, AND PETER HUMPHRIES
}

AbStract. Consider a semiclassical Hamiltonian

$$
H_{V, h}:=h^{2} \Delta+V-E
$$

where $h>0$ is a semiclassical parameter, $\Delta$ is the positive Laplacian on $\mathbb{R}^{d}, V$ is a smooth, compactly supported central potential function and $E>0$ is an energy level. In this setting the scattering matrix $S_{h}(E)$ is a unitary operator on $L^{2}\left(\mathbb{S}^{d-1}\right)$, hence with spectrum lying on the unit circle; moreover, the spectrum is discrete except at 1 .

We show under certain additional assumptions on the potential that the eigenvalues of $S_{h}(E)$ can be divided into two classes: a finite number $\sim c_{d}(R \sqrt{E} / h)^{d-1}$, as $h \rightarrow 0$, where $B(0, R)$ is the convex hull of the support of the potential, that equidistribute around the unit circle, and the remainder that are all very close to 1 . Semiclassically, these are related to the rays that meet the support of, and hence are scattered by, the potential, and those that do not meet the support of the potential, respectively.

A similar property is shown for the obstacle problem in the case that the obstacle is the ball of radius $R$.

\section{INTRODUCTION}

In this paper we consider the scattering matrix for a semiclassical potential scattering problem with spherical symmetry on $\mathbb{R}^{d}, d \geq 2$. Let $V$ be a smooth, compactly supported potential function which is central, i.e. $V(x)$ depends only on $|x|$. We consider the Hamiltonian

$$
H_{V, h}:=h^{2} \Delta+V-E
$$

where $\Delta=-\sum_{i=1}^{d} \partial_{i}^{2}$ is the positive Laplacian on $\mathbb{R}^{d}, E>0$ is a positive constant (energy) and $h>0$ is a semiclassical parameter. At the end of the introduction we will reduce to the case $E=1$.

The scattering matrix $S_{h}(E)$ for this Hamiltonian can be defined in terms of the asymptotics of generalized eigenfunctions of $H_{V, h}$ as follows. For each function $q_{i n} \in C^{\infty}\left(\mathbb{S}^{d-1}\right)$, there is a unique solution to $H_{V, h} u=0$ of the form

$$
u=r^{-(d-1) / 2}\left(e^{-i \sqrt{E} r / h} q_{\text {in }}(\omega)+e^{+i \sqrt{E} r / h} q_{\text {out }}(-\omega)\right)+O\left(r^{-(d+1) / 2}\right),
$$

as $r \rightarrow \infty$, see e.g. [17. Here $q_{\text {out }} \in C^{\infty}\left(\mathbb{S}^{d-1}\right)$. The map $q_{\text {in }} \mapsto e^{i \pi(d-1) / 2} q_{\text {out }}$ is by definition the scattering matrix $S_{h}(E)$. The factor $e^{i \pi(d-1) / 2}$ is chosen so that this 'stationary' definition agrees with time-dependent definitions (see e.g. 21] or [25]), and is such that

The first author was partially supported by an NSF postdoctoral fellowship. The authors would like to thank Hamid Hezari, Volker Schlue and Steve Zelditch for very helpful conversations. The third author acknowledges the support of the Australian Research Council through a Future Fellowship FT0990895 and Discovery Grant DP1095448. 
the scattering matrix for the potential $V \equiv 0$ is the identity map. It is standard that the scattering matrix $S_{h}(E)$ is a unitary operator on $L^{2}\left(\mathbb{S}^{d-1}\right)$ for every $h>0$, and that, for the potentials under consideration, $S_{h}(E)$ - Id is compact. It follows that the spectrum lies on the unit circle, consists only of eigenvalues, and is discrete except at 1 . It is therefore possible to count the number of eigenvalues of $S_{h}(E)$ in any closed interval of the unit circle not containing 1 . In fact, semiclassically (i.e. as $h \rightarrow 0$ ) we are able to separate the spectrum of $S_{h}(E)$ into two parts. One is associated to the rays that meet the support of the potential; to leading order in $h$ there are $c_{d}(R \sqrt{E} / h)^{d-1}$ of these eigenvalues, $c_{d}=2 /(d-1)$ !, and the other part is associated to the rays that do not meet the support of the potential. Those eigenvalues corresponding to rays that do not meet the support are close to 1 , as one should expect, since the eigenvalues of the zero potential are all 1 - see Proposition 1.5 below. The other eigenvalues are affected by the potential, and we can ask whether these 'nontrivial' eigenvalues are asymptotically equidistributed on the unit circle. Indeed Steve Zelditch posed this question to one of the authors several years ago.

Before stating the main result, we discuss further the scattering matrix in the case of central potentials. In this case the eigenfunctions of the scattering matrix are spherical harmonics and the generalized eigenfunctions then take the form $u=r^{-(d-2) / 2} f(r) Y_{l}^{m}$, where

$$
\left(-\partial_{r}^{2}-\frac{1}{r} \partial_{r}+\frac{(l+(d-2) / 2)^{2}}{r^{2}}+\frac{V(r)-E}{h^{2}}\right) f=0 .
$$

Here

$$
f(r)=H_{l+(d-2) / 2}^{(1)}(r \sqrt{E} / h)+c(l) H_{l+(d-2) / 2}^{(2)}(r \sqrt{E} / h) \text { for } r>R,
$$

where the $H_{\nu}^{(i)}$ are the standard Hankel functions, [1. With our normalization, $S_{h} Y_{l}^{m}=$ $c(l) Y_{l}^{m}$ with $c(l)$ from (1.4) In particular, the eigenvalue of $Y_{l}^{m}$ is independent of $m$. We write the eigenvalue corresponding to $Y_{l}^{m}$ in the form $e^{i \beta_{l, h}}$. The quantities $\beta_{l, h} / 2$ are called 'phase shifts.' See e.g. 21] for a review of these facts.

We now discuss conditions on the potentials in the main theorems. These conditions are dynamical conditions, i.e. conditions on the Hamiltonian dynamical system determined by the symbol of $H_{V, h}$. As usual in microlocal analysis we refer to the classical trajectories of this system as bicharacteristics. We first define the interaction region

$$
\mathcal{R}:=\{x: V(|y|)<E \text { for all }|y|>|x|\} .
$$

This is the region of $x$-space accessible by bicharacteristics coming from infinity. Notice that for central potentials this region takes the form

$$
\mathcal{R}=\left\{|x| \geq r_{0}\right\} \text { where } r_{0}=\inf _{r \geq 0}\{s>r \Rightarrow V(s)<E\} .
$$

The first condition is

$$
V \text { is nontrapping at energy } E \text { in the interaction region. }
$$

That is, $x$ tends to infinity along every bicharacteristic in $\mathcal{R}$ both forwards and backwards in time.

The second condition concerns the scattering angle. Let $R$ be such that $B(0, R)$ is the smallest ball containing the support of $V$, i.e.

$$
B(0, R)=\operatorname{chsupp} V \text {, the convex hull of the support of } \mathrm{V} \text {. }
$$

We recall (see Section 2 for definitions and details) that for a central potential, the scattering angle $\Sigma(\alpha)$ is a function only of the angular momentum $\alpha$ and measures the difference 
between the incident and final directions of the trajectory (which are well-defined, since the motion is free for $|x|>R$ - see [21]. The scattering angle is zero for all trajectories with $\alpha>R$. Our second condition is that

$$
\text { the number of zeroes of } \Sigma^{\prime}(\alpha)=\frac{d \Sigma}{d \alpha}(\alpha) \text { in }[0, R) \text { is finite. }
$$

Then our main results are

Theorem 1.1. Let $R$ be as in (1.8), and assume that $V \in C_{c}^{\infty}\left(\mathbb{R}^{d}\right)$ is central and satisfies condition (1.7). Define the real-valued function $G(\alpha), \alpha \in \mathbb{R}$, by

$$
\frac{d G}{d \alpha}(\alpha)=\Sigma(\alpha), \quad G(\alpha)=0 \text { for } \alpha \geq R
$$

where $\Sigma$ is the scattering angle function in 2.5). Then the following approximation on each eigenvalue $e^{i \beta_{l, h}}$ of $S_{h}$ is valid:

(i) If the dimension $d$ is even, then there exists $C=C(d)$ such that, for all $l \in \mathbb{N}$ satisfying lh $\leq R$, we have an estimate

$$
\left|e^{i \beta_{l, h}}-\exp \left\{\frac{i}{h}\left(G\left(\left(l+\frac{d-2}{2}\right) h\right)\right)\right\}\right| \leq C h .
$$

(ii) If the dimension $d>2$ is odd, then for any $\epsilon>0$ there exists $C=C(\epsilon, d)$ such that (1.11) holds whenever $\alpha=l h \geq \epsilon$ is distance at least $\epsilon$ from the set

$$
\left\{\alpha: \Sigma(\alpha) \in\{\pi k\}_{k \in \mathbb{Z}}\right\} .
$$

Theorem 1.2. Let $R$ be as in (1.8), and assume that $V \in C_{c}^{\infty}\left(\mathbb{R}^{d}\right)$ is central and satisfies conditions (1.7) and (1.9). Then as $h \downarrow 0$, we consider the eigenvalues $e^{i \beta_{l, h}}$ for which $l \leq R \sqrt{E} / h$, counted with multiplicity $p_{d}(l)=\operatorname{dim} \operatorname{ker}\left(\Delta_{\mathbb{S}^{d-1}}-l(l+d-2)\right)$. There are $2(R \sqrt{E} / h)^{d-1} /(d-1) !+O\left(h^{-(d-2)}\right)$ of these, and they equidistribute around the unit circle, meaning that

$$
\sup _{0 \leq \phi_{0}<\phi_{1} \leq 2 \pi}\left|\frac{N\left(\phi_{0}, \phi_{1}\right)}{2(R \sqrt{E} / h)^{d-1} /(d-1) !}-\frac{\phi_{1}-\phi_{0}}{2 \pi}\right| \rightarrow 0 \text { as } h \downarrow 0,
$$

where $N\left(\phi_{0}, \phi_{1}\right)$ is the number of $\beta_{l, h}$ with $l \leq R \sqrt{E} / h$ and $\phi_{0} \leq \beta_{l, h} \leq \phi_{1}(\bmod 2 \pi)$, counted with multiplicity.

Remark 1.3. The approximation 1.11 for the phase shifts can be found in physics textbooks; see for example [15, Sect. 126] or [19, Equation (18.11), Section 18.2]; it can be derived readily from the WKB approximation applied to (1.3). However, no error estimate is claimed in either of these sources. We have not been able to find any rigorous bounds on the WKB approximation of the phase shifts in any previous literature, so we believe the bound (1.11) to be new.

Remark 1.4. Many potentials satisfy conditions 1.7$)$ and 1.90 - see Section 5 .

We also show

Proposition 1.5. Let $V$ and $R$ be as in Theorem 1.1, and let $\kappa \in(0,1)$. The eigenvalues $e^{i \beta_{l, h}}$ for $l \geq\left(R \sqrt{E}+h^{\kappa}\right) / h$ satisfy

$$
\left|e^{i \beta_{l, h}}-1\right|=O\left(h^{\infty}\right), \quad h \rightarrow 0 .
$$


Here and below, $O\left(h^{\infty}\right)$ denotes a quantity that is bounded by $C_{N} h^{N}$ for all $N$ and some $C_{N}>0$.

Remark 1.6. The methods of [20, Section 4] show that for a 'black box perturbation' of the Laplacian on $\mathbb{R}^{d}$, at most $O\left(h^{-(d-1)}\right)$ eigenvalues of the scattering matrix are essentially different from 1.

Note that the number of eigenvalues not covered by Theorem 1.1 and Proposition 1.5 is $o\left(h^{1-d}\right)$, and hence cannot affect the equidistribution properties. Hence we get the following equidistribution result for the full sequence of eigenvalues of $S_{h}$.

Corollary 1.7. Suppose that $V$ satisfies conditions (1.7) and (1.9). Then for each $\epsilon>0$, we have

$$
\sup _{\epsilon \leq \phi_{0}<\phi_{1} \leq 2 \pi-\epsilon}\left|\frac{\tilde{N}\left(\phi_{0}, \phi_{1}\right)}{2(R \sqrt{E} / h)^{d-1} /(d-1) !}-\frac{\phi_{1}-\phi_{0}}{2 \pi}\right| \rightarrow 0 \text { as } h \downarrow 0,
$$

where $\tilde{N}\left(\phi_{0}, \phi_{1}\right)$ is the number of $\beta_{l, h}$ (with no condition on l), counted with multiplicity, satisfying $\phi_{0}<\beta_{l, h}<\phi_{1}(\bmod 2 \pi)$.

Results directly analogous to those for semiclassical potentials are also true in the case of scattering by a disk of radius $R$ centered at the origin. The scattering matrix in this case can be defined similarly; given any function $q_{\text {in }} \in C^{\infty}\left(\mathbb{S}^{d-1}\right)$, there is a unique solution $u$ to the equation $\left(\Delta-k^{2}\right) u=0$ such that ${ }^{1}$

$$
\begin{aligned}
u= & r^{-(d-1) / 2}\left(e^{-i k r} q_{\text {in }}(\omega)+e^{+i k r} q_{\text {out }}(-\omega)\right)+O\left(r^{-(d+1) / 2}\right), \quad r \rightarrow \infty \\
& \left.u\right|_{|x|=R} \equiv 0
\end{aligned}
$$

The scattering matrix $S_{k}$ is again defined $q_{\text {in }} \mapsto e^{i \pi(d-1) / 2} q_{\text {out }}$, and the standard facts about the operator $S_{h}$ also hold for $S_{k}$. As above, the spherical harmonics diagonalize the scattering matrix. We write $S_{k} Y_{l}^{m}=e^{i x_{l, k}} Y_{l}^{m}$. We will prove

Theorem 1.8. As $k \rightarrow \infty$, the eigenvalues $e^{i x_{l, k}}$ of $S_{k}$ satisfy

$$
\left|e^{i x_{l, k}}-e^{i\left(k G_{b}((l+(d-2) / 2) / k)+\pi / 2\right)}\right| \leq C k^{-1 / 2}, \quad \frac{l}{k} \leq R-k^{-1 / 3}
$$

for some uniform $C=C(d)$, where $\Sigma_{b}(\alpha):=-2 \cos ^{-1}(\alpha / R)$ is the scattering angle for the ball and $G_{b}$ is defined by

$$
G_{b}(\alpha)=-\int_{\alpha}^{R} \Sigma_{b}\left(\alpha^{\prime}\right) d \alpha^{\prime}=2 \sqrt{R^{2}-\alpha^{2}}-2 \alpha \cos ^{-1}(\alpha / R) .
$$

The points $e^{i x_{l, k}}$ for which $l \leq R k$, counted with multiplicity

$$
p_{d}(l)=\operatorname{dim} \operatorname{ker}\left(\Delta_{\mathbb{S}^{d-1}}-l(l+d-2)\right),
$$

equidistribute around $\mathbb{S}^{1}$ in the sense of Theorem 1.2. In fact, we have the stronger statement

$$
\sup _{0 \leq \phi_{0}<\phi_{1} \leq 2 \pi}\left|\frac{N\left(\phi_{0}, \phi_{1}\right)}{2(R k)^{d-1} /(d-1) !}-\frac{\phi_{1}-\phi_{0}}{2 \pi}\right|=O\left(k^{-1 / 3}\right) \text { as } k \rightarrow \infty .
$$

\footnotetext{
${ }^{1}$ Here we prefer to use non-semiclassical notation where the energy level is $k^{2}$, as is traditional in obstacle scattering literature. The variable $k$ here corresponds to $1 / h$ above, when the energy level $E=1$.
} 
As far as we are aware, the present paper is the first in the mathematical literature to deal with the question of the equidistribution of phase shifts over the unit circle. However, there are a number of previous studies of high-energy or semiclassical asymptotics of eigenvalues of the scattering matrix. The relation between the sojourn time and high-frequency asymptotics of the scattering matrix was observed in classical papers by Guillemin [9, Majda [16] and Robert-Tamura [22. Melrose and Zworski [18] showed that for fixed $h>0$ the absolute scattering matrix for a Schrödinger operator on a scattering, or asymptotically conic, manifold is an FIO associated to the geodesic flow on the manifold at infinity for time $\pi$. Alexandrova 2] studied the scattering matrix for a nontrapping semiclassical Schrödinger operator, and showed that localized to finite frequency, it is a semiclassical FIO associated to the limiting Hamilton flow relation at infinity, which includes the behavior of the Hamilton flow in compact sets. A more global description was given in Hassell-Wunsch [10] where the semiclassical asymptotics of the scattering matrix were unified with the singularities of the scattering matrix at fixed frequency (i.e. the Melrose-Zworski result [18). These results are explained in Sections 2 and 3 below.

Asymptotics of phase shifts, i.e. the logarithms of the eigenvalues of the scattering matrix, were analysed by Birman-Yafaev [3, 4, 5, 6, Sobolev-Yafaev [24, Yafaev 26] and more recently Bulger-Pushnitski [7]. In [24, an asymptotic form $V \sim c r^{-\alpha}, \alpha>2$ was assumed and asymptotics of the individual phase shifts as well as the scattering cross section were obtained. In this paper, the strength of the potential and the energy were allowed to vary independently, so that the result includes the semiclassical limit as in the present paper. In the other papers listed above, the context was scattering theory for a fixed potential. In this setting, the scattering matrix $S(\lambda)$ tends in operator norm to the identity as $\lambda \rightarrow \infty$ so the phase shifts tend to zero uniformly. The asymptotics of individual phase shifts for a fixed energy, and also the high-energy asymptotics, were analyzed.

In the 1990s Doron and Smilansky studied the pair correlation for phase shifts, in particular proposing that the pair correlations should behave statistically similarly to the (conjectural) pair correlations for eigenfunctions of a closed quantum system: that is, the pair correlations for chaotic systems should be the same as for certain ensembles of random matrices, while for completely integrable systems, they should be Poisson distributed (the 'Berry-Tabor conjecture'); see for example [8]. In [28], Zelditch and Zworski analyzed the pair correlation function for eigenvalues of the scattering matrix associated to a rotationally invariant surface with a conic singularity and a cylindrical end. They showed that a full measure set of a 2-parameter family of such surfaces obeyed Poisson statistics, agreeing with Smilansky's conjecture.

In a different setting Zelditch [27] analyzed quantized contact transformations, which are families of unitary maps on finite dimensional spaces with dimension $N \rightarrow \infty$. He proved under the assumption that the set of periodic points of the transformation has measure zero, that the eigenvalues of these unitary operators becomes equidistributed as $N \rightarrow \infty$. After reading a draft of the current paper, Zelditch pointed out to the authors that a similar strategy could be used in the context of semiclassical potential scattering to prove equidistribution. In fact, this strategy is likely to be a more direct approach to proving equidistribution than the one we employ here. On the other hand, our approach has several advantages: it also gives approximations to the individual phase shifts, up to an $O(h)$ error (see Theorem 1.1), and in addition appears to be a better method for obtaining a rate of equidistribution, as in Theorem 1.8 above.

In future work, we plan to treat non-central potentials (or perhaps, following the suggestion of one of the referees, black box perturbations of the free Laplacian - see [23, 20]) as 
well as non-compactly supported potentials. In the latter case, 24 gives some indication of what to expect; in particular, the scaling with $h$ cannot be the same as in the compactly supported case.

Reduction to $E=1$ : In light of 1.3 and 1.4 ,

$$
S_{h, V}(E)=S_{\widetilde{h}, \widetilde{V}}(1),
$$

where $\widetilde{h}=h / \sqrt{E}$ and $\widetilde{V}=V / E$. Here $S_{h, V}(E)$ denotes the scattering matrix of $h^{2} \Delta+V-E$, and $S_{\widetilde{h}, \widetilde{V}}(1)$ denotes the scattering matrix of $\widetilde{h}^{2} \Delta+\widetilde{V}-1$. For the remainder of the paper, we assume without loss of generality that $E=1$.

\section{DYNAMiCS}

We now review some standard material on Hamiltonian dynamics for central potentials. Consider first the case the dimension $d=2$.

The classical Hamiltonian corresponding to our quantum system is

$$
|\xi|^{2}+V(r)-1
$$

or in polar coordinates, using $(r, \varphi)$ and dual coordinates $(\rho, \eta)$,

$$
H=\rho^{2}+\frac{\eta^{2}}{r^{2}}+V(r)-1,
$$

and the Hamilton equations of motion are

$$
\begin{aligned}
\dot{r} & =2 \rho & \dot{\varphi} & =2 \frac{\eta}{r^{2}} \\
\dot{\rho} & =-V^{\prime}(r)+2 r^{-3} \eta^{2} & \dot{\eta} & =0 .
\end{aligned}
$$

The invariance of the Hamiltonian under rotations is reflected in the conservation of angular momentum $\eta=2 r^{2} \dot{\varphi}$. For a given bicharacteristic, this is the minimum value of $r$ along the free $(V \equiv 0)$ bicharacteristic that agrees with the given one as $t \rightarrow-\infty$ (we could just as well take $t \rightarrow+\infty$ since it is a conserved quantity).

Notice that in the case of general dimension $d$, each bicharacteristic lies entirely in a two-dimensional subspace, so the above discussion in fact includes the general case.

The scattering matrix is related to the asymptotic properties of the bicharacteristic flow. Geometrically this information is contained in a submanifold

$$
L \subset T^{*} \mathbb{S}^{d-1} \times T^{*} \mathbb{S}^{d-1} \times \mathbb{R}
$$

that we define now. Returning to the case of general dimension $d$, we identify $\mathbb{S}^{d-1}$ with the unit sphere in $\mathbb{R}^{d}$ and identify the cotangent space $T_{\omega}^{*} \mathbb{S}^{d-1}$ with the orthogonal hyperplane $\omega^{\perp}$ to $\omega$. Given $\omega$ and $\eta \in T_{\omega}^{*} \mathbb{S}^{d-1}$, take the unique bicharacteristic ray whose projection $x_{\omega, \eta}(t)$ to $\mathbb{R}^{d}$ is given by $\eta+t \omega$ for $t<<0$. Define $\left(\omega^{\prime}, \eta^{\prime}\right)$ by

$$
\begin{aligned}
& \omega^{\prime}(\omega, \eta)=\lim _{t \rightarrow \infty} x(t) /|x(t)| \\
& \eta^{\prime}(\omega, \eta)=\lim _{t \rightarrow \infty} x(t)-\left\langle x(t), \omega^{\prime}\right\rangle \omega^{\prime}
\end{aligned}
$$

and $\tau(\omega, \eta)$ to be the sojourn time or time delay for $\gamma$; this is by definition the limit

$$
\lim _{a \rightarrow \infty} t_{1}(a)-t_{2}(a)-2 a=\tau(\omega, \eta),
$$




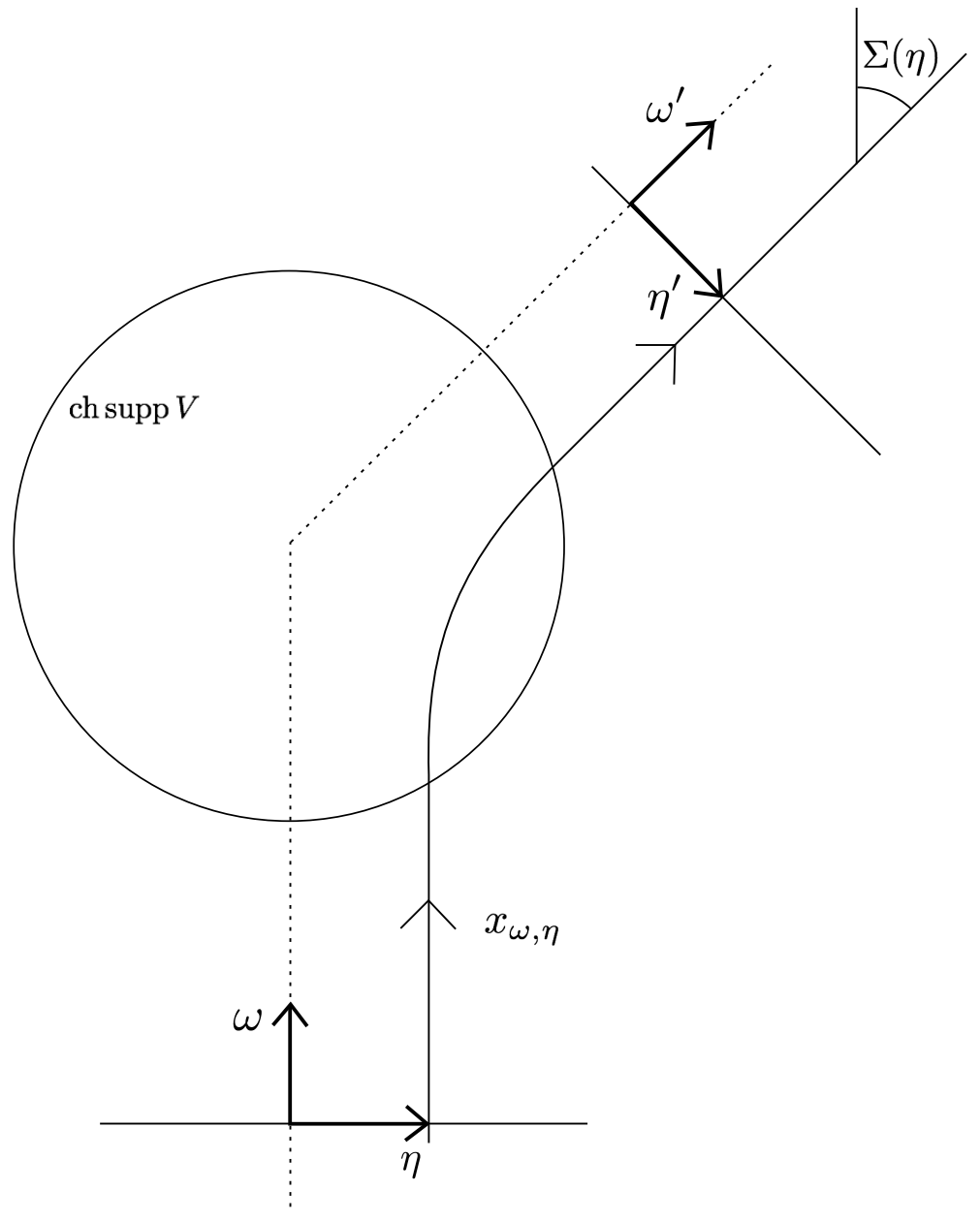

Figure 1. Here $x_{\omega, \eta}$ is the classical trajectory equal to $\eta+t \omega$ for $t<<0$. The scattering angle $\Sigma(\eta)$ is the angle between the outgoing direction $\omega^{\prime}$ and the incoming direction $\omega$. Note that $|\eta|=\left|\eta^{\prime}\right|$ by conservation of angular momentum since the potential $V$ is central, $V=V(r)$.

where $t_{1}(a)$ is the smallest time, $t$, for which $r(t)=a$ and $t_{2}(a)$ is the largest. We then define $L$ to be the submanifold

$$
L:=\left\{\left(\omega, \eta, \omega^{\prime}(\omega, \eta),-\eta^{\prime}(\omega, \eta), \tau\right) \cdot\right\}
$$

As shown in [10, $L$ is a Legendrian submanifold of $T^{*} \mathbb{S}^{d-1} \times T^{*} \mathbb{S}^{d-1} \times \mathbb{R}$ with respect to the contact form $\chi+\chi^{\prime}-d \tau$, where $\chi$ is the standard contact form on $T^{*} \mathbb{S}^{d-1}$, given in any local coordinates $x$ and dual coordinates $\xi$ by $\xi \cdot d x$. Note that the projection of $L$ to $T^{*} \mathbb{S}^{d-1} \times T^{*} \mathbb{S}^{d-1}$ is Lagrangian with respect to the standard symplectic form. Indeed it is the graph of a symplectic transformation $(\omega, \eta) \mapsto\left(\omega^{\prime}, \eta^{\prime}\right)$, and the scattering matrix is a semiclassical Fourier integral operator associated to this symplectic graph [2], [10]. The sojourn time, however, carries extra information and is directly related to high-energy scattering asymptotics as observed in [16, [9], [10]. 
The previous paragraph applies to any potential, central or not. We return to the case of a central potential $V$, for which, as observed above, the dynamics take place in a twodimensional subspace, so we can assume $d=2$ without loss of generality. In that case we use the angular variables $\varphi, \varphi^{\prime}$ in dimension $d=2$ instead of $\omega, \omega^{\prime}$ above. Consider a bicharacteristic $\gamma$ with angular momentum $\eta \in \mathbb{R}$, initial direction $\varphi \in \mathbb{S}^{1}$ and final direction $\varphi^{\prime} \in \mathbb{S}^{1}$. The scattering angle $\Sigma(\eta)$ determined by $V$ is, by definition, the angle between the initial and final directions of $\gamma$, normalized so that $\Sigma$ is continuous and $\Sigma(\eta)=0$ for $\eta>R$, i.e.

$$
\Sigma(\eta)=\varphi^{\prime}(\varphi, \eta)-\varphi, \quad \Sigma(\eta)=0 \text { for } \eta>R .
$$

See Figure 1. Note that $\Sigma$ is independent of $\varphi$ by rotational invariance of $V$. In the central case, there is a standard expression for $\Sigma(\eta)$ in terms of the potential (see for example [19. Section 5.1]), that we now derive. Indeed, if $V$ is central and non-trapping at energy 1 , then along a bicharacteristic, the functions $\rho$ and $\dot{\rho}$ do not have simultaneous zeros. For if there were such a time, and the value of $r$ at this time were $r_{0}$, then $r(t) \equiv r_{0}$ would be a bicharacteristic, contradicting the non-trapping assumption. Hence the zeros of the function $1-\eta^{2} / r^{2}-V(r)$ are simple on the region of interaction 1.5. Given a fixed bicharacteristic, let $r_{m}$ be the minimum value of $r$; note that $r_{m}$ is a function only of the angular momentum $\eta$. We denote the derivative of $r_{m}$ with respect to $\eta$ by $r_{m}^{\prime}(\eta)$. By symmetry, $r_{m}$ is the unique value of $r$ along the trajectory at which $\rho=0$ and $\dot{\rho}>0$, so we can divide the bicharacteristic 'in half,' and consider only times when $r>r_{m}$ and $\rho \geq 0$. For such times $r$ is a strictly monotone function of $t$, and we have

$$
\frac{d \varphi}{d r}=\frac{d \varphi}{d t} \frac{d t}{d r}=\frac{\eta}{r^{2}} \frac{1}{\rho}=\frac{\eta}{r^{2} \sqrt{1-\eta^{2} / r^{2}-V(r)}} .
$$

By the simplicity of the zeros in the denominator, we can integrate to obtain, for $\eta>0$,

$$
\Sigma(\eta)=\pi-2 \int_{r_{m}}^{\infty} \frac{\eta}{r^{2} \sqrt{1-\eta^{2} / r^{2}-V(r)}} d r .
$$

The sojourn time is also independent of $\varphi$, and we write

$$
T(\eta)=\tau(\varphi, \eta)
$$

Notice that both $\Sigma$ and $T$ depend only on $\eta$ in the central case. The fact that $L$ in $(2.4)$ is Legendrian then implies the following relation between these functions:

$$
d \tau=\eta \cdot\left(d \varphi-d \varphi^{\prime}\right) \Longrightarrow \frac{d}{d \eta} T(\eta)=-\eta \frac{d}{d \eta} \Sigma(\eta)
$$

Remark 2.1. Notice that the ambiguity of $\Sigma$ modulo $2 \pi$ is eliminated by our convention that $\Sigma(\eta)=0$ for $\eta>R$. We point out that by reflection symmetry, we have $\Sigma(\eta)=-\Sigma(-\eta)$ modulo $2 \pi$, but it might not be the case that $\Sigma(\eta)=-\Sigma(-\eta)$ on the nose: this will happen if and only if $\Sigma(0)=0$, which will be the case if and only if the interaction region is the whole of $\mathbb{R}^{d}$. However, we always have $\Sigma^{\prime}(\eta)=\Sigma^{\prime}(-\eta)$, which shows that $T^{\prime}(\eta)$ is an odd function, and hence $T(\eta)$ is even in $\eta$.

\section{Asymptotic For the eigenvalues of $S_{h}$}

In this section we prove Theorem 1.1, that is, the error bound 1.11 for the asymptotics of the eigenvalues $e^{i \beta_{l, h}}$ of $S_{h}$. To do this, we will use a Fourier integral approach. One could also directly attack 1.3 using ODE methods; see Remark 3.3 for further discussion on this point. 
We use the fact, proven in [10, 2], that the integral kernel of $S_{h}$ is an oscillatory integral associated (in a manner we describe directly) to the Legendre submanifold $L$ in 2.4. To be precise, the Schwartz kernel of $S_{h}$ can be decomposed following [10, Prop. 15] (with minor changes in notation) as

$$
S_{h}=K_{1}+K_{2}+K_{3}
$$

with the $K_{i}$ as follows.

Fix $R_{2}>R_{1}>R$. First, $K_{2}$ is a pseudodifferential operator of order zero (both in the sense of semiclassical order and differential order), microsupported in $\left\{|\eta|>R_{1}\right\}$, hence taking the form in local coordinates $z$ on $\mathbb{S}^{d-1}$

$$
(2 \pi h)^{-(d-1)} \int e^{i\left(z-z^{\prime}\right) \cdot \zeta / h} b(z, \zeta, h) d \zeta
$$

for some smooth symbol $b(z, \zeta, h)$ equal to zero for $|\zeta|_{g(z)}<R_{1}$ where $|\cdot|_{g(z)}$ is the standard norm on $T_{z}^{*} \mathbb{S}^{d-1}$. This reflects the fact that the Legendrian submanifold $L$ in $(2.4)$ is the diagonal relation $\omega=\omega^{\prime}, \eta=-\eta^{\prime}, \tau=0$ for $|\eta|,\left|\eta^{\prime}\right|>R$, to which pseudodifferential operators are associated. Moreover, $K_{2}$ is microlocally equal to the identity for $|\eta|>R_{2}$, i.e. $b=1+O\left(h^{\infty}\right)$ for $|\zeta|_{g}>R_{2}$. Indeed, the full symbol (up to $O\left(h^{\infty}\right)$ ) of the scattering matrix is determined by transport equations along the rays with $|\eta|>R$. Since these transport equations are identical to those for the zero potential, the scattering matrix in this microlocal region is microlocally identical to that for the zero potential, which is the identity operator.

Next, $K_{1}$ is a semiclassical Fourier integral operator of semiclassical order 0 with compact microsupport in $\left\{|\eta|<R_{2}\right\}$. That is, $K_{1}$ is given by a sum of terms taking the form in local coordinates

$$
K_{1}\left(\omega, \omega^{\prime}, h\right)=h^{-(d-1) / 2-N / 2} \int_{\mathbb{R}^{N}} e^{i \Phi\left(\omega, \omega^{\prime}, v\right) / h} a\left(\omega, \omega^{\prime}, v, h\right) d v
$$

with respect to a suitable phase function $\Phi$ and smooth compactly supported function $a$. Here the phase function parametrizes $L$ locally, meaning

(1) On the set Crit $\Phi:=\left\{\left(\omega, \omega^{\prime}, v\right): D_{v} \Phi\left(\omega, \omega^{\prime}, v\right)=0\right\}, D_{\omega, \omega^{\prime}, v} \Phi$ has rank $N$. This implies that

$$
L(\Phi):=\left(\omega, D_{\omega} \Phi\left(\omega, \omega^{\prime}, v\right), \omega^{\prime}, D_{\omega^{\prime}} \Phi\left(\omega, \omega^{\prime}, v\right), \Phi\left(\omega, \omega^{\prime}, v\right)\right)
$$

is a smooth submanifold.

(2) $L(\Phi)=L$ at points for which $a \neq O\left(h^{\infty}\right)$.

By $K_{1}$ having compact microsupport in the set $\left\{|\eta|<R_{2}\right\}$, we mean specifically that if $\left(\omega, \eta, \omega^{\prime}, \eta^{\prime}, \tau\right) \in L$ has $|\eta|=|\eta|^{\prime}>R_{2}$ and $\left(\omega, \omega^{\prime}, v\right) \in \operatorname{Crit}(\Phi)$ with $\left(D_{\omega, \omega^{\prime}} \Phi\left(\omega, \omega^{\prime}, v_{i}\right), \Phi\right)=$ $\left(\eta, \eta^{\prime}, \tau\right)$, then $a\left(\omega, \omega^{\prime}, v, h\right)=O\left(h^{\infty}\right)$ in a neighbourhood of $\left(\omega, \omega^{\prime}, v\right)$.

Finally, $K_{3}$ is a kernel in $\dot{C}^{\infty}\left(\mathbb{S}^{d-1} \times \mathbb{S}^{d-1} \times\left[0, h_{0}\right)\right)$, i.e. smooth and vanishing to all orders at $h=0$.

For the proof of Theorem 1.1 we need to know the principal symbol of $K_{1}$ as a semiclassical FIO. By (3.2), the canonical relation of $K_{1}, C$, is the projection of $L$ off the $\mathbb{R}$ factor, i.e. onto $T^{*} \mathbb{S}^{d-1} \times T^{*} \mathbb{S}^{d-1}$. Precisely, with notation as in 2.4,

$$
C=\left\{\left(\omega, \eta, \omega^{\prime},-\eta^{\prime}\right)\right\}
$$

Lemma 3.1. The Maslov bundle of the canonical relation $C$ of the FIO $K_{1}$ is canonically trivial, and with respect to this canonical trivialization, the principal symbol of $K_{1}$ is equal 
to 1, as a multiple of the Liouville half-density on $C$ coming from either the left or right projection of $C$ to $T^{*} \mathbb{S}^{d-1}$. That is to say,

$$
\sigma\left(K_{1}\right)\left(\omega, \eta, \omega^{\prime},-\eta^{\prime}\right)=|d \omega d \eta|^{1 / 2}=\left|d \omega^{\prime} d \eta^{\prime}\right|^{1 / 2},
$$

for $\left(\omega, \eta, \omega^{\prime},-\eta^{\prime}\right) \in C$ such that $|\eta| \leq R_{1}$. (The two half-densities in (3.4) are equal on $C$ since $C$ is a Lagrangian submanifold.)

Proof. Consider first the Maslov bundle of $C$. Notice that $C$ is almost the same as $L$; in fact, it is given by

$$
C=\left\{\left(\omega, \eta, \omega^{\prime},-\eta^{\prime}\right) \mid \exists \tau \operatorname{such} \text { that }\left(\omega, \eta, \omega^{\prime}, \eta^{\prime}, \tau\right) \in L\right\} .
$$

Since $C$ is a canonical graph (i.e. the graph of a symplectomorphism), associated to the scattering relation as in (2.2), it projects diffeomorphically to $T^{*} \mathbb{S}^{d-1}$ via both the left and right projections, and the lift of Liouville measure on $T^{*} \mathbb{S}^{d-1}$ via the left projection agrees with the lift via the right projection (since $C$ is a Lagrangian submanifold of $T^{*} \mathbb{S}^{d-1} \times$ $T^{*} \mathbb{S}^{d-1}$ and the Liouville measure can be expressed in terms of the symplectic form $T^{*} \mathbb{S}^{d-1}$ ), providing a canonical half-density on $C$. We also note that the scattering relation is the identity whenever $|\eta| \geq R$ since then the corresponding bicharacteristic is not affected by the potential. Therefore, over this part of $C$ there is a canonical trivialization of the Maslov bundle. Since the Maslov bundle is flat, we can use parallel transport to extend this to a global trivialization: in fact, in the case $d=2$, the space $T^{*} \mathbb{S}^{1}$ retracts to $\mathbb{S}^{d-1} \times\{\eta>R\}$, while for $d \geq 3, T^{*} \mathbb{S}^{d-1}$ is simply connected, hence in either case parallel transport provides an unambiguous trivialization.

We now consider the principal symbol of the scattering matrix. The scattering matrix may be viewed as a 'boundary value' (after removing a vanishing factor and an oscillatory term) of the Poisson operator, as in [10, Section 7.7 and Section 15]. The principal symbol of the scattering matrix is correspondingly derived from the principal symbol of the Poisson operator. The principal symbol of the Poisson operator is real: it solves a real transport equation with initial condition 1 . Therefore, the principal symbol of the scattering matrix is real, up to Maslov factors, i.e. it is a real number times an eighth root of unity. On the other hand, unitarity of the scattering matrix shows that the principal symbol lies on the unit circle (as a multiple of the canonical half-density); hence it is an eighth root of unity. Finally, the principal symbol of the scattering matrix is equal to 1 for $|\eta| \geq R$, since here the scattering matrix is microlocally equal to the scattering matrix for the zero potential, which is certainly equal to 1 . Since the principal symbol is smooth, is restricted to eighth roots of unity, and is 1 for $|\eta| \geq R$, it follows that the principal symbol is equal to 1 everywhere.

Proof of Theorem 1.1: First we reduce the problem to the cases $d=2$ and $d=3$ as follows. Writing $\beta_{l, h, d}$ for the eigenvalue $\beta_{l, h}$ in dimension $d$, observe that by (1.3),

$$
\beta_{l, h, d+2 k}=\beta_{l+k, h, d} \text { for } d \geq 2, k \geq 0 .
$$

It follows that for $d \geq 4$ even, we have $\beta_{l, h, d}=\beta_{l+(d-2) / 2, h, 2}$ and for $d \geq 5$ odd, we have $\beta_{l, h, d}=\beta_{l+(d-3) / 2, h, 3}$.

Consider the case dimension $d=2$. For any smooth function $G: \mathbb{R} \longrightarrow \mathbb{R}$, the function

$$
\Phi\left(\varphi, \varphi^{\prime}, v\right)=\left(\varphi-\varphi^{\prime}\right) v+G(v),
$$

parametrizes the Legendrian (see $(3.2)$ )

$$
L(\Phi):=\left\{\left(\varphi, \eta, \varphi^{\prime}, \eta^{\prime}, \tau\right) \mid \eta=v=-\eta^{\prime}, \varphi^{\prime}-\varphi=\frac{d G}{d v}(v), \tau=-v \frac{d G}{d v}(v)+G(v)\right\} .
$$


With $G$ as in 1.10 , this gives an explicit global parametrization of the Legendrian submanifold $L$ in $(2.4)$ if we take $\varphi \in[0,2 \pi], \varphi^{\prime} \in \mathbb{R}$. In this case the relation between $\tau$ and $\Sigma$ given by the last equation in (3.7) is

$$
\tau=-\eta \Sigma(\eta)+G(\eta) \Longrightarrow \frac{d}{d \eta} \tau=-\eta \frac{d}{d \eta} \Sigma(\eta)
$$

in agreement with (2.8). Therefore, plugging (3.6) into (3.1), the operator $K_{1}$ takes the form

$$
K_{1}\left(\varphi, \varphi^{\prime}, h\right)=(2 \pi h)^{-1} \int_{\mathbb{R}} e^{i\left(\left(\varphi-\varphi^{\prime}\right) v+G(v)\right) / h} a\left(\varphi-\varphi^{\prime}, v, h\right) d v, \quad \varphi \in[0,2 \pi], \varphi^{\prime} \in \mathbb{R},
$$

where $a$ is smooth and supported in $|v| \leq R_{2}$. Notice that we may assume that $a$ depends only on $\left(\varphi-\varphi^{\prime}, v, h\right)$ since the scattering matrix and the phase function both have this property.

Now we obtain an expression for the eigenvalue $e^{i \beta_{l, h}}$ of the scattering matrix $S_{h}$ on $Y_{l}=(2 \pi)^{-1 / 2} e^{i l \varphi}$ using

$$
e^{i \beta_{l}, h}=\left\langle S_{h} Y_{l}, Y_{l}\right\rangle=\left\langle K_{1} Y_{l}, Y_{l}\right\rangle+\left\langle K_{2} Y_{l}, Y_{l}\right\rangle+\left\langle K_{3} Y_{l}, Y_{l}\right\rangle
$$

Clearly $\left\langle K_{3} Y_{l}, Y_{l}\right\rangle=O\left(h^{\infty}\right)$. Consider the $K_{1}$ term. Writing $l=\alpha / h$ gives

$$
\left\langle K_{1} Y_{l}, Y_{l}\right\rangle=(2 \pi h)^{-1}(2 \pi)^{-1} \int_{\mathbb{R}} \int_{0}^{2 \pi} \int_{\mathbb{R}} e^{i\left(\left(\varphi-\varphi^{\prime}\right) v+G(v)-\alpha\left(\varphi-\varphi^{\prime}\right)\right) / h} a\left(\varphi-\varphi^{\prime}, v, h\right) d v d \varphi d \varphi^{\prime} .
$$

Changing integration variables to $\left(\varphi, \tilde{\varphi}=\varphi-\varphi^{\prime}\right)$, the kernel is independent of the first of these variables, so that integrating in it simply removes the factor $2 \pi$. We are left with

$$
\left\langle K_{1} Y_{l}, Y_{l}\right\rangle=(2 \pi h)^{-1} \int_{\mathbb{R}} \int_{\mathbb{R}} e^{i(\tilde{\varphi} v+G(v)-\alpha \tilde{\varphi}) / h} a(\tilde{\varphi}, v, h) d v d \tilde{\varphi}
$$

The phase is stationary at the point $v=\alpha,-\tilde{\varphi}=G^{\prime}(v)=\Sigma(v)$ and the stationary phase lemma shows that the integral is equal to

$$
\left\langle K_{1} Y_{l}, Y_{l}\right\rangle=e^{i G(\alpha) / h} a(-\Sigma(\alpha), \alpha, 0)+O(h)
$$

(noting that the Hessian of the phase function has determinant 1 and signature 0 ).

Next we write

$$
\left\langle K_{2} Y_{l}, Y_{l}\right\rangle=(2 \pi h)^{-1}(2 \pi)^{-1} \int e^{i\left(\varphi-\varphi^{\prime}\right) v / h} b(\varphi, v, h) e^{-i \alpha\left(\varphi-\varphi^{\prime}\right) / h} d v d \varphi d \varphi^{\prime}
$$

Here, the phase is stationary when $\alpha=v$. However, $b$ is supported where $|v| \geq R_{1}>R$ while $\alpha \leq R$ by hypothesis, so there are no stationary points on the support of the integrand. It follows that $\left\langle K_{2} Y_{l}, Y_{l}\right\rangle=O\left(h^{\infty}\right)$. Thus by (3.8)

$$
e^{i \beta_{l, h}}=e^{i G(\alpha) / h} a(-\Sigma(\alpha), \alpha, 0)+O(h) .
$$

The principal symbol of $K_{1}$ as an FIO is given as a multiple of the Liouville half-density on $T^{*} \mathbb{S}^{1},|d \varphi d \eta|^{1 / 2}$, by [11, Section 3]

$$
\sigma\left(K_{1}\right)(\varphi, \eta, \varphi+\Sigma(\eta),-\eta)=a(-\Sigma(\eta), \eta, 0)|d \varphi d \eta|^{1 / 2}
$$

Indeed, the density $d_{C}$ defined on page 143 of that paper equals $|d \varphi d \eta|$, where we used coordinates $(x, \theta)=\left(\varphi, \varphi^{\prime}, v\right)$. The principal symbol is the image of the map from $C$ to $\Lambda$ defined immediately following the definition of $d_{C}$, in the notation of that paper. In the notation of the current paper, $C=\operatorname{Crit}(\Phi)$ and $\Lambda$ is the projection of the Legendrian $L$ 
onto the first four coordinates, i.e. it is $C$ from 3.3 . It follows from equation (3.11) and equation (3.4) that

$$
a(-\Sigma(\eta), \eta, 0)=1
$$

Combining (3.12) and 3.9 , we see that

$$
e^{i \beta_{l, h}}=e^{i G(\alpha) / h}+O(h)
$$

establishing (1.11).

We proceed to the case $d=3$. In this case, we will obtain the eigenvalue $e^{i \beta_{l, h}}$ by pairing the scattering matrix $S_{h}$ with the highest weight spherical harmonics $Y_{l}^{l}$. These concentrate along a great circle $\gamma$, which we parametrize by arclength, $\varphi \in[0,2 \pi]$. Choose Euclidean coordinates in $\mathbb{R}^{3}$ so that the two-plane spanned by $\gamma$ is the plane $x_{3}=0$. Then $Y_{l}^{l}=c_{l}\left(x_{1}+i x_{2}\right)^{l}$ where $c_{l}$ is a normalization factor, equal to $(2 \pi)^{-1 / 2}(\pi l)^{1 / 4}\left(1+O\left(l^{-1}\right)\right)$. Let $\theta$ be the spherical coordinate equal to the angle with the positive $x_{3}$ axis. Then we can write

$$
Y_{l}^{l}(\varphi, \theta)=c_{l} e^{i l \varphi}(\sin \theta)^{l}=c_{l} e^{i l \varphi} e^{-l g(\theta)}
$$

where $g(\theta)=-\log \sin \theta=(\theta-\pi / 2)^{2} / 2+O\left((\theta-\pi / 2)^{4}\right)$.

In particular, expression (3.14 shows (and it is in any case well known) that the $Y_{l}^{l}$ concentrate semiclassically at the set $\{\theta=\pi / 2, \zeta=0, \sigma=\alpha\}$ where $l=\alpha / h+O(1)$. Here we use coordinates $(\sigma, \zeta)$ dual to $(\varphi, \theta)$. To compute the pairing $(3.8)$ with $Y_{l}^{l}$ replacing $Y_{l}$, we first need to determine an oscillatory integral expression for $K_{1}$ that is valid in this microlocal region. (Note that the $K_{2}$ and $K_{3}$ terms give an $O\left(h^{\infty}\right)$ contribution as before.) So choose $\alpha_{0}$ distance $\geq \epsilon$ from the set 1.12 . As we will see, it suffices to find a local parametrization of $L$ in a neighbourhood of

$$
\left\{\theta=\theta^{\prime}=\pi / 2, \zeta=\zeta^{\prime}=0, \sigma=-\sigma^{\prime}=\alpha_{0}, \varphi-\varphi^{\prime}=\Sigma\left(\alpha_{0}\right)\right\} ;
$$

this is the set of incoming and outgoing data of bicharacteristics with angular momentum $\alpha_{0}$ (see Section 2) which remain in the $x_{3}=0$ plane. To define this parametrization, we consider first a parametrization in two dimensions locally near a bicharacteristic with angular momentum $\eta=\alpha$. As we have seen such a two dimensional parametrization is $\left(\varphi-\varphi^{\prime}\right) v+G(v)$, for $v$ close to $\alpha$. We note that when $v=\alpha, \varphi^{\prime}-\varphi=\Sigma(\alpha)$, and we can write it in the form

$$
\varphi^{\prime}-\varphi= \pm \operatorname{dist}\left(\varphi, \varphi^{\prime}\right)+2 \pi k
$$

for some integer $k$ (recalling that the distance $\operatorname{dist}\left(\varphi, \varphi^{\prime}\right)$ lies strictly between 0 and $\pi$ ). We now claim that a suitable phase function is

$$
\Phi\left(\omega, \omega^{\prime}, v\right)=\left(\mp \operatorname{dist}\left(\omega, \omega^{\prime}\right)-2 \pi k\right) v+G(v),
$$

where $v \in \mathbb{R}$ is localized near $\alpha_{0}, G(v)$ is as in 1.10 , and the sign $\mp$ and the value of $k$ agree with the two-dimensional case. Indeed, on each two-plane, if we use spherical coordinates $(\bar{\varphi}, \bar{\theta})$ adapted to that 2-plane then the form of the phase function agrees by construction with the two-dimensional phase function and therefore parametrizes that part of $L$ associated to that 2-plane (since the dynamics on each 2-plane is identical to the $d=2$ dynamics), that is, the subset (in the coordinates adapted to that 2-plane, indicated by a bar)

$$
\left\{\bar{\theta}=\bar{\theta}^{\prime}=\pi / 2, \bar{\zeta}=\bar{\zeta}^{\prime}=0, \bar{\varphi}^{\prime}-\bar{\varphi}=\Sigma(\alpha), \bar{\sigma}=-\bar{\sigma}^{\prime}=\alpha, \tau=T(\alpha)\right\} .
$$

We now observe that we can eliminate $k$ by redefining $G(v)$ locally to be $G(v)+2 \pi k v$, which only has the irrelevant effect of changing $\Sigma$ by $2 \pi k$ (notice also that this does not 
affect the eigenvalue formula involving $e^{i G(l h) / h}$ in the statement of Theorem 1.1. From here on we only work with the $+\operatorname{sign}$ in $(3.15)$, i.e. the $-\operatorname{sign}$ in $(3.16)$, and $k=0$. Notice that this means that $0<\varphi^{\prime}-\varphi<\pi$ and $0<\Sigma(\alpha)<\pi$, i.e. $\sin \Sigma(\alpha)>0$. Returning to our spherical coordinates associated to the 2-plane $x_{3}=0$, we can use the spherical cosine law applied to the spherical triangle with vertices $(\varphi, \theta),\left(\varphi^{\prime}, \theta^{\prime}\right)$, and the pole $x_{3}=1$ :

$$
\cos \operatorname{dist}\left((\varphi, \theta),\left(\varphi^{\prime}, \theta^{\prime}\right)\right)=\cos \left(\varphi-\varphi^{\prime}\right) \sin \theta \sin \theta^{\prime}+\cos \theta \cos \theta^{\prime}
$$

to write

$$
\Phi\left(\varphi, y, \varphi^{\prime}, y, v\right)=-\cos ^{-1}\left(\cos \left(\varphi-\varphi^{\prime}\right) \sin \theta \sin \theta^{\prime}+\cos \theta \cos \theta^{\prime}\right) v+G(v) .
$$

We can then write in these coordinates

$$
\begin{aligned}
L=\{ & \left(\varphi, \theta, \varphi^{\prime}, \theta^{\prime}, \sigma, \zeta, \sigma^{\prime}, \zeta^{\prime}, \tau\right) \mid \\
\sigma & =\partial_{\varphi} \Phi=-\frac{v}{\sin \operatorname{dist}\left(\omega, \omega^{\prime}\right)}\left(\sin \left(\varphi-\varphi^{\prime}\right) \sin \theta \sin \theta^{\prime}\right) \\
& =\partial_{\theta} \Phi=\frac{v}{\sin \operatorname{dist}\left(\omega, \omega^{\prime}\right)}\left(\cos \left(\varphi-\varphi^{\prime}\right) \cos \theta \sin \theta^{\prime}-\sin \theta \cos \theta^{\prime}\right) \\
\sigma^{\prime} & =\partial_{\varphi^{\prime}} \Phi=\frac{v}{\sin \operatorname{dist}\left(\omega, \omega^{\prime}\right)}\left(\sin \left(\varphi-\varphi^{\prime}\right) \sin \theta \sin \theta^{\prime}\right) \\
\zeta^{\prime} & =\partial_{\theta^{\prime}} \Phi=\frac{v}{\sin \operatorname{dist}\left(\omega, \omega^{\prime}\right)}\left(\cos \left(\varphi-\varphi^{\prime}\right) \cos \theta^{\prime} \sin \theta-\sin \theta^{\prime} \cos \theta\right) \\
\tau & \left.=\operatorname{dist}\left(\omega, \omega^{\prime}\right) v+G(v)\right\} \quad \text { where } \operatorname{dist}\left(\omega, \omega^{\prime}\right)=G^{\prime}(v) .
\end{aligned}
$$

(Notice that by direct inspection we see that this agrees with (3.17) when $\theta=\theta^{\prime}=\pi / 2$, since then $\cos \theta=\cos \theta^{\prime}=0$ and $\operatorname{dist}\left(\omega, \omega^{\prime}\right)=\varphi^{\prime}-\varphi=\Sigma(\alpha)$ and so $\sigma=v=\alpha$.)

The scattering matrix, microlocalized to this region of phase space, will then take the form

$$
(2 \pi h)^{-3 / 2} \int e^{i \Phi\left(\omega, \omega^{\prime}, v\right) / h} a\left(\omega, \omega^{\prime}, v, h\right) d v .
$$

In terms of this parametrization the principal symbol of (3.20), say where both $\omega$ and $\omega^{\prime}$ lie near the great circle $\gamma$ and hence where we can use coordinates $\left(\varphi, \theta, \varphi^{\prime}, \theta^{\prime} ; \sigma, \zeta, \sigma^{\prime}, \zeta^{\prime}, \tau\right)$, is given at the point $(\varphi, \pi / 2, \varphi+\Sigma(\alpha) h, \pi / 2, \alpha, 0,-\alpha, 0, \tau(\alpha))$ by [11]

$$
a(\varphi, \pi / 2, \varphi+\Sigma(\alpha), \pi / 2, \alpha, 0) e^{-i \pi / 4}|d s d \theta d \sigma d \zeta|^{1 / 2}\left|\operatorname{det} \frac{\partial\left(\varphi, \theta, \sigma, \zeta, d_{v} \Phi\right)}{\partial\left(\varphi, \theta, \varphi^{\prime}, \theta^{\prime}, v\right)}\right|^{-1 / 2}
$$

where the $e^{-i \pi / 4}$ is a Maslov factor; see Remark 3.2 for more discussion about this. We need to compute the determinant above. We can disregard the repeated coordinates $(\varphi, \theta)$ and compute, using (3.19),

$$
\operatorname{det} \frac{\partial\left(\sigma, \zeta, d_{v} \Phi\right)}{\partial\left(\varphi^{\prime}, \theta^{\prime}, v\right)}=\operatorname{det}\left(\begin{array}{ccc}
0 & 0 & -1 \\
0 & \frac{-v}{\sin \left(\varphi^{\prime}-\varphi\right)} & 0 \\
-1 & 0 & G^{\prime \prime}(v)
\end{array}\right)=\frac{v}{\sin \left(\varphi^{\prime}-\varphi\right)}=\frac{\alpha}{\sin \Sigma(\alpha)} \text { at } \theta=\theta^{\prime}=\frac{\pi}{2} \text {. }
$$

It follows that the principal symbol is

$$
a(\varphi, \pi / 2, \varphi+\Sigma(\alpha), \pi / 2, \alpha, 0) e^{-i \pi / 4}\left(\frac{\alpha}{\sin \Sigma(\alpha)}\right)^{-1 / 2}|d s d \theta d \sigma d \zeta|^{1 / 2} .
$$


Then by equation (3.4

$$
a(\varphi, \pi / 2, \varphi+\Sigma(\alpha), \pi / 2, \alpha, 0) e^{-i \pi / 4}=\left(\frac{\alpha}{\sin \Sigma(\alpha)}\right)^{1 / 2}
$$

We next write the contribution of $K_{1}$ to the expression 3.8 for the eigenvalue $e^{i \beta_{l, h}}$. Writing $l=\alpha / h$ and using (3.14) we get

$$
\begin{aligned}
\left\langle K_{1} Y_{l}^{l}, Y_{l}^{l}\right\rangle=(2 \pi h)^{-3 / 2} \int e^{i \Phi\left(\varphi, \theta, \varphi^{\prime}, \theta^{\prime}, v\right) / h} e^{-i \alpha\left(\varphi-\varphi^{\prime}\right) / h}\left(\frac{\alpha}{\pi h}\right)^{1 / 2}(2 \pi)^{-1} & \\
& \times e^{-\alpha g(\theta) / h} e^{-\alpha g\left(\theta^{\prime}\right) / h} a\left(\varphi, \theta, \varphi^{\prime}, \theta^{\prime}, v, h\right) d s d \varphi^{\prime} d \theta d \theta^{\prime} d v(1+O(h))
\end{aligned}
$$

Here the factors $(\alpha / \pi h)^{1 / 2}(2 \pi)^{-1}$ are to normalize the functions $Y_{l}^{l}$ in $L^{2}$. We will analyze this using the stationary phase lemma with complex phase function, see e.g. [12, thm. 7.7.5]. Here the phase is

$$
\Psi\left(\varphi, \theta, \varphi^{\prime}, \theta^{\prime}, v\right)=\Phi-\alpha\left(\varphi-\varphi^{\prime}\right)+i \alpha\left(g(\theta)+g\left(\theta^{\prime}\right)\right) .
$$

Notice that the integrand as a function of $\left(\varphi, \varphi^{\prime}\right)$ depends only on $\varphi-\varphi^{\prime}$ by the rotational invariance of the scattering matrix, and the form of the $Y_{l}^{l}$ which take the form $e^{i l \varphi}$ times a function of $\theta$. We change variable to $(\varphi, \tilde{\varphi}), \tilde{\varphi}=\varphi-\varphi^{\prime}$ and integrate out the variable $\varphi$, giving us a factor of $2 \pi$. Then $\Psi$ has nondegenerate stationary points in the remaining variables $\left(\tilde{\varphi}, \theta, \theta^{\prime}, v\right)$. The imaginary part of the phase is stationary only at $\theta=\theta^{\prime}=\pi / 2$, while stationarity of the real part requires that $v=\alpha$ and $-\tilde{\varphi}=G^{\prime}(v)=\Sigma(\alpha)$. The stationary phase lemma then gives us that 3.25 is equal to

$$
\begin{aligned}
& 2 \pi\left((2 \pi h)^{-3 / 2}\left(\frac{\alpha}{\pi h}\right)^{1 / 2}(2 \pi)^{-1}\right)(2 \pi h)^{2} \\
& \quad \times\left(e^{i G(\alpha) / h} \frac{1}{\operatorname{det}\left(-i D^{2} \Psi\right)^{1 / 2}} a(\varphi, \pi / 2, \varphi+\Sigma(\alpha), \pi / 2, \alpha, 0)+O(h)\right) .
\end{aligned}
$$

Here, to keep track of constants, we have written out all constants in (3.25); the first $2 \pi$ comes from the integral in $\varphi$ and the $(2 \pi h)^{2}$ comes from the leading term in stationary phase in the four variables $\left(\tilde{\varphi}, \theta, \theta^{\prime}, v\right)$. Simplifying the constants and using 3.24 this is equal to

$$
\left(\frac{2 \alpha^{2}}{\sin \Sigma(\alpha)}\right)^{1 / 2}\left(\frac{e^{i G(\alpha) / h}}{\left(\operatorname{det}-i D^{2} \Psi\right)^{1 / 2}}+O(h)\right) .
$$

We will show that, in the above expression

$$
\operatorname{det}-i D^{2} \Psi(\varphi, 0, \varphi+\Sigma(\alpha), 0, \alpha)=\frac{2 i \alpha^{2}}{\sin \Sigma(\alpha)} e^{-i \Sigma(\alpha)} .
$$

Accepting this for the moment, we obtain from 3.27

$$
e^{i \beta_{l, h}}=e^{i G(\alpha) / h} e^{i \Sigma(\alpha) / 2}+O(h) .
$$

Since $\Sigma(\alpha)=G^{\prime}(\alpha)$ and $\alpha=l h$, this can be written

$$
e^{i \beta_{l, h}}=e^{i G((l+1 / 2) h) / h}+O(h)
$$

completing the proof of Theorem 1.1 
It remains to prove the formula for the Hessian in 3.29 . First we notice that when $\theta=\theta^{\prime}=\pi / 2$ we have, using the formula in 3.18 for the distance function, in the coordinates $\left(\tilde{\varphi}, \theta, \theta^{\prime}\right)$

$$
D^{2} \operatorname{dist}(\varphi, \pi / 2, \varphi+\Sigma(\alpha), \pi / 2)=\left(\begin{array}{ccc}
0 & 0 & 0 \\
0 & \cot \Sigma(\alpha) & -\csc \Sigma(\alpha) \\
0 & -\csc \Sigma(\alpha) & \cot \Sigma(\alpha)
\end{array}\right)
$$

From this 3.16 and 3.26 , we conclude that in the $\left(v, \widetilde{\varphi}, y, y^{\prime}\right)$ coordinates

$$
D^{2} \Psi=\left(\begin{array}{cccc}
G^{\prime \prime}(v) & 1 & 0 & 0 \\
1 & 0 & 0 & 0 \\
0 & 0 & -\alpha \cot \Sigma(\alpha)+i \alpha & \alpha \csc \Sigma(\alpha) \\
0 & 0 & \alpha \csc \Sigma(\alpha) & -\alpha \cot \Sigma(\alpha)+i \alpha
\end{array}\right)
$$

Thus

$$
\operatorname{det}-i D^{2} \Psi=-\alpha^{2}\left(\cot ^{2} \Sigma(\alpha)-2 i \cot \Sigma(\alpha)-1-\csc ^{2} \Sigma(\alpha)\right)=\frac{2 i \alpha^{2}}{\sin \Sigma(\alpha)} e^{-i \Sigma(\alpha)}
$$

and 3.29 holds.

Remark 3.2. The Maslov factor in 3.21 and (3.24) arises as follows. First, Lemma 3.1 shows that the Maslov bundle over $L$ is canonically trivial. However, unlike in the case $d=2$, there is a nontrivial Maslov factor from comparing our phase function $\Phi$ above to one — let us call it $\tilde{\Phi}$ — that agrees with the canonical phase function, i.e. the pseudodifferential phase function, for $|\eta| \geq R$. By [11, Theorem 3.2.1], the principal symbol written relative to $\Phi$ contains the Maslov factor $e^{i \pi \sigma / 4}$ where $\sigma$ is the difference of signatures,

$$
\sigma=\operatorname{sgn} D_{v v}^{2} \Phi-\operatorname{sgn} D_{\tilde{w} \tilde{w}}^{2} \tilde{\Phi}
$$

where $\tilde{w}=\left(\tilde{w}_{1}, \tilde{w}_{2}\right)$ are the phase variables for $\tilde{\Phi}$. A tedious computation shows that $\sigma=-1$, leading to the Maslov factor in (3.21) and (3.24). (We remark that since $\Phi$ depends on one phase variable and $\tilde{\Phi}$ on two phase variables, by [11, Equation (3.2.12)] $\sigma$ is odd, so the Maslov factor cannot vanish in this case.) Of course, the Maslov factors are irrelevant to the question of equidistribution, but they are relevant to the question of determining the eigenvalues modulo $O(h)$.

Proof of Proposition 1.5. In view of the remarks in the proof of Theorem 1.1. specifically equation (3.5), it is only necessary to do this in the cases $d=2$ and $d=3$. For definiteness, we write down the proof for $d=3$; it is similar, and in fact simpler, for $d=2$. Consider a spherical harmonic $Y_{l}^{l}$ with $h l \geq R+h^{\kappa}$, where $\kappa<1$. The eigenvalue $e^{i \beta_{l, h}}$ is given by 3.8 with $Y_{l}^{l}$ replacing $Y_{l}$.

First assume that $h l \geq R^{\prime}>R$. Then the $K_{1}$ term in 3.8 will be $O\left(h^{\infty}\right)$ (for a suitable decomposition of $S_{h}=K_{1}+K_{2}+K_{3}$ as above, with $R_{2}<R^{\prime}$ ), so we only have to consider the $K_{2}$ term. This is given by a pseudodifferential operator with symbol equal to $1+O\left(h^{\infty}\right)$, so the $\left\langle Y_{l}^{l}, K_{2} Y_{l}^{l}\right\rangle$ term is equal to $1+O\left(h^{\infty}\right)$, proving the Proposition in this case.

Next assume that $R+h^{\kappa} \leq h l \leq R_{1}^{\prime}$. For $R^{\prime}<R_{1}$, the $K_{2}$ term in (3.8) will be $O\left(h^{\infty}\right)$ (for some other decomposition of $S_{h}$, with $R_{1}>R^{\prime}$ ), so we only need to consider the $K_{1}$ term. That is, it remains to show that

$$
\left\langle\left(K_{1}-\mathrm{Id}\right) Y_{l}^{l}, Y_{l}^{l}\right\rangle=O\left(h^{\infty}\right) \text { for } R+h^{\kappa} \leq h l
$$


Using as above polar coordinates $(\varphi, \theta)$ on $S^{2}$ with dual coordinate $(\sigma, \zeta)$, we find a phase function $\Psi$ for $K_{1}$ that parametrizes $L$ microlocally in the region

$$
\left\{|(\sigma, \zeta)|_{g} \geq R-\delta\right\}
$$

for fixed small $\delta>0$. Indeed, since $L$ is given by the diagonal relation

$$
\left\{\varphi=\varphi^{\prime}, \theta=\theta^{\prime}, \sigma=-\sigma^{\prime}, \zeta=-\zeta^{\prime}, \tau=0\right\} \text { for }\left\{|(\sigma, \zeta)|_{g} \geq R\right\},
$$

it follows that the functions $\left(\varphi, \theta, \sigma^{\prime}, \zeta^{\prime}\right)$ furnish local coordinates on the Legendrian $L$ for $\left\{|(\sigma, \zeta)|_{g} \geq R\right\}$ and therefore, by continuity, for $\left\{|(\sigma, \zeta)|_{g} \geq R-\delta\right\}$ for some small $\delta>0$. It then follows from [13, Theorem 21.2.18] that $L$ can be parametrized by a phase function of the form

$$
-\varphi^{\prime} v-\theta^{\prime} w+H(\varphi, \theta, v, w)
$$

Since $K_{1}$ is pseudodifferential for $\left|\left(\sigma^{\prime}, \zeta^{\prime}\right)\right|_{g}^{2}=\zeta^{\prime 2}+\left(\sin \theta^{\prime}\right)^{-2}{\sigma^{\prime 2}}^{2} \geq R^{2}$ (i.e. $L$ satisfies 3.33 ), we have

$$
v \geq R \sin \theta^{\prime} \Longrightarrow H=\varphi v+\theta w \text { and } b=1+O\left(h^{\infty}\right)
$$

Thus

$$
\begin{array}{r}
\left\langle\left(K_{1}-\mathrm{Id}\right) Y_{l}^{l}, Y_{l}^{l}\right\rangle=\int\left(e^{i\left(-\varphi^{\prime} v-\theta^{\prime} w+H(\varphi, \theta, v, w)\right) / h} b\left(\varphi^{\prime}, \theta^{\prime}, v, w, h\right)-e^{i\left(\left(\varphi-\varphi^{\prime}\right) v+\left(\theta-\theta^{\prime}\right) w\right) / h}\right) \\
\times c_{l} e^{i \alpha\left(g(\theta)+g\left(\theta^{\prime}\right)\right) / h} \frac{d \varphi d \theta d \varphi^{\prime} d \theta^{\prime} d v d w}{(2 \pi h)^{3}}+O\left(h^{\infty}\right),
\end{array}
$$

As above we have written $l=\alpha / h$; hence $\alpha>R+h^{\kappa}$.

We insert cutoff functions by writing

$$
1=\chi\left(\frac{v-R \sin \theta^{\prime}}{h^{\kappa}}\right)+(1-\chi)\left(\frac{v-R \sin \theta^{\prime}}{h^{\kappa}}\right)
$$

where $\chi(t)$ is supported in $t \leq 1 / 2$, equal to 1 for $t \leq 1 / 4$. With the cutoff $\chi$ inserted, the phase function is nonstationary on the support of the integrand, since stationarity requires that $v=\alpha$. It follows that we can integrate by parts arbitrarily many times, using the fact that the differential operator

$$
\frac{1}{v-\alpha} \frac{h}{i} \frac{\partial}{\partial \varphi^{\prime}}
$$

leaves both exponential factors invariant; doing this gains a factor of $h^{1-\kappa}$ each time since $\alpha-v \geq h^{\kappa} / 2$ on the support of the integrand. Thus the $\chi$ term is $O\left(h^{\infty}\right)$.

With the cutoff $1-\chi$ inserted, we write the integral in (3.34) in the form

$$
\begin{array}{r}
\int e^{i\left(\left(\varphi-\varphi^{\prime}\right) v+\left(\theta-\theta^{\prime}\right) w\right) / h}\left(e^{i(H(\varphi, \theta, v, w)-\varphi v-\theta w) / h} b\left(\varphi^{\prime}, \theta^{\prime}, v, w, h\right)-1\right) \\
\times c_{l}(1-\chi)\left(\frac{v-R \sin \theta^{\prime}}{h^{\kappa}}\right) e^{i \alpha\left(g(\theta)+g\left(\theta^{\prime}\right)\right) / h} \frac{d \varphi d \theta d \varphi^{\prime} d \theta^{\prime} d v d w}{(2 \pi h)^{3}} .
\end{array}
$$

We claim that the factor

$$
\left(e^{i(H(\varphi, \theta, v, w)-\varphi v-\theta w) / h} b\left(\varphi^{\prime}, \theta^{\prime}, v, w, h\right)-1\right) \times(1-\chi)\left(\frac{v-R \sin \theta^{\prime}}{h^{\kappa}}\right)
$$


is $O\left(h^{\infty}\right)$. In fact, the term in the large brackets is $O\left(h^{\infty}\right)$ for $v \geq R \sin \theta^{\prime}$, while if $v \leq$ $R \sin \theta^{\prime}$, then the $1-\chi$ term vanishes identically. It follows that the $1-\chi$ term is also $O\left(h^{\infty}\right)$, completing the proof of Proposition 1.5 .

Remark 3.3. The reader may wonder whether a direct ODE attack on 1.3 might be simpler and more straightforward than our FIO approach to this problem, given that our approach relies on 10, Theorem 15.6], which in turn rests on a significant amount of machinery. By contrast the WKB expansion for the solution yields the approximation for the eigenvalues in Theorem 1.1 in a straightforward fashion. However, although it is not hard to write down a WKB approximation to the solutions of (1.3), it seems (to the authors) that proving rigorous error bounds for such WKB expansions is rather subtle. The problem is that to prove such bounds, one must solve away the error term, that is, get good estimates on the solution to the imhomogeneous ODE where the inhomogeneous term (the error term when the WKB approximation is substituted into $(1.3 p)$ is $O\left(h^{N}\right)$ for some sufficiently large $N$. Notice that the ODE (1.3) might have several turning points, and the desired solution is governed by a boundary condition $f^{\prime}(0)=0$ at the origin, so one needs to understand the behaviour of the solution passing through possibly several turning points. Since the solutions may grow exponentially in the non-interaction region, this does not seem to be easy or straightforward, and we are not aware of anywhere in the literature where this has been written down. Carrying out this procedure would certainly be a worthwhile enterprise, but we have chosen instead to build on the above-mentioned theorem about the semiclassical scattering matrix which is already available in the literature.

Other features recommend the FIO approach in this context. First, the relationship between the scattering angle and the phase-shifts is made transparent here, or at least it is 'reduced' to the fact that the integral kernel of $S_{h}$ is a semi-classical FIO whose canonical relation 'contains' the scattering angle, while on the other hand from the formula produced by the WKB this relationship is not immediately apparent. More importantly, FIO methods will be essential in treating the noncentral case, which we intend to do in future work, and the symmetric case under consideration is a situation in which $S_{h}$ can be understood almost explicitly.

\section{EQUidistribution}

If $\omega=\left\{e^{2 \pi i x_{1}}, \ldots, e^{2 \pi i x_{K}}\right\}$ is any set of $K$ points on $\mathbb{S}^{1}$, then the discrepancy $D(\omega)$ is defined by

$$
D(\omega):=\sup _{0 \leq \phi_{0}<\phi_{1} \leq 2 \pi}\left|\frac{N\left(\phi_{0}, \phi_{1} ; \omega\right)}{K}-\frac{\phi_{1}-\phi_{0}}{2 \pi}\right|,
$$

where $N\left(\phi_{0}, \phi_{1} ; \omega\right)$ is the number of points in $\omega$ with argument in $\left[\phi_{0}, \phi_{1}\right]$ (modulo $2 \pi$ ), counted with multiplicity. We state the following lemma in slightly more generality then is necessary for semiclassical potentials so that we may apply it without significant modification to the case of scattering by the disk.

Lemma 4.1. Let $G:[0, R) \longrightarrow \mathbb{R}$ be smooth and assume that

$$
\left\{\alpha: G^{\prime \prime}(\alpha)=0\right\} \text { is finite in }[0, R) .
$$

Consider the points $x_{l k}$ on the unit circle

$$
\mathcal{E}_{h}=\left\{x_{l k}:=\exp (i G(l h) / h): 0 \leq l h<R, k=1, \ldots, p_{d}(l)\right\},
$$

included according to multiplicity. Here $p_{d}(l)=\operatorname{dim} \operatorname{ker}\left(\Delta_{\mathbb{S}^{d-1}}-l(l+d-2)\right)$. 
Then the sets $\mathcal{E}_{h}$ equidistribute as $h \rightarrow 0$. That is, the discrepancy satisfies

$$
\lim _{h \rightarrow 0} D\left(\mathcal{E}_{h}\right)=0 \text {. }
$$

To apply the lemma to the eigenvalues of the scattering matrix $S_{h}$, we must show that they still equidistribute despite satisfying only the weaker asymptotic condition in Theorem 1.1 .

Proposition 4.2. Let $S \subset[0, R]$ be a finite set and let

$$
\widetilde{\mathcal{E}}_{h}=\left\{\widetilde{x}_{l k}: 0 \leq l h \leq R, k=1, \ldots, p_{d}(l)\right\}
$$

be a collection of points on $\mathbb{S}^{1}$ (included according to multiplicity), such that for any $\epsilon>0$, if $l$ satisfies $\operatorname{dist}(l h, S) \geq \epsilon$ then

$$
\left.\widetilde{x}_{l k}=\exp i(G(l h) / h)+E(l, h)\right)
$$

where $|E(l, h)|<C(\epsilon) h$. Then, if $G$ satisfies condition 4.2 in Lemma 4.1.

$$
\lim _{h \rightarrow 0} D\left(\widetilde{\mathcal{E}_{h}}\right)=0 .
$$

We will use the following notation. With any set $S$ as above, let

$$
\mathcal{E}_{h}(\epsilon):=\mathcal{E}_{h} \cap\left\{x_{l k}: \operatorname{dist}(l h, S) \geq \epsilon\right\} \quad \text { and } \quad \widetilde{\mathcal{E}}_{h}(\epsilon):=\widetilde{\mathcal{E}}_{h} \cap\left\{\widetilde{x}_{l k}: \operatorname{dist}(l h, S) \geq \epsilon\right\},
$$

always understood to include points according to multiplicity.

Proof of Proposition 4.2 assuming Lemma 4.1: The error bound $|E(l, h)|<C(\epsilon) h$ shows that, for every $\epsilon>0$, there is a constant $C=C(\epsilon, S)>0$ so that

$N\left(\phi_{0}+C h, \phi_{1}-C h ; \mathcal{E}_{h}(\epsilon)\right) \leq N\left(\phi_{0}, \phi_{1} ; \widetilde{\mathcal{E}_{h}}(\epsilon)\right) \leq N\left(\max \left(\phi_{0}-C h, 0\right), \min \left(\phi_{1}+C h, 2 \pi\right) ; \mathcal{E}_{h}(\epsilon)\right)$.

Dividing through by $2(R / h)^{d-1} /(d-1)$ !, subtracting $\left(\phi_{1}-\phi_{0}\right) / 2 \pi$, and taking $h$ small gives

$$
\begin{aligned}
\left|\frac{N\left(\phi_{0}, \phi_{1} ; \widetilde{\mathcal{E}_{h}}(\epsilon)\right)}{2(R / h)^{d-1} /(d-1) !}-\frac{\phi_{1}-\phi_{0}}{2 \pi}\right| \leq \max \left\{\left|\frac{N\left(\phi_{0}+C h, \phi_{1}-C h ; \mathcal{E}_{h}(\epsilon)\right)}{2(R / h)^{d-1} /(d-1) !}-\frac{\phi_{1}-\phi_{0}}{2 \pi}\right|,\right. & \left.\left|\frac{N\left(\phi_{0}-C h, \phi_{1}+C h ; \mathcal{E}_{h}(\epsilon)\right)}{2(R / h)^{d-1} /(d-1) !}-\frac{\phi_{1}-\phi_{0}}{2 \pi}\right|\right\} \\
& \leq D\left(\mathcal{E}_{h}(\epsilon)\right)+(1+C(\epsilon)) O(h)+O(\epsilon),
\end{aligned}
$$

uniformly in $h$ and $\epsilon$, where for the second inequality we used

$$
\left|\mathcal{E}_{h}(\epsilon)\right|=\frac{2 R^{d-1}}{h^{d-1}(d-1) !}(1+O(h)+O(\epsilon)),
$$

where $\left|\mathcal{E}_{h}(\epsilon)\right|$ is the number of points in $\mathcal{E}_{h}(\epsilon)$. Similarly, $D\left(\mathcal{E}_{h}\right)=D\left(\mathcal{E}_{h}(\epsilon)\right)+O(h)+O(\epsilon)$ for $h, \epsilon$ small, and the same is true for $\widetilde{\mathcal{E}}_{h}$. Thus

$$
\left|\frac{N\left(\phi_{0}, \phi_{1} ; \widetilde{\mathcal{E}_{h}}\right)}{2(R / h)^{d-1} /(d-1) !}-\frac{\phi_{1}-\phi_{0}}{2 \pi}\right| \leq D\left(\mathcal{E}_{h}\right)+(1+C(\epsilon)) O(h)+O(\epsilon) .
$$

Thus

$$
\limsup _{h \rightarrow 0} D\left(\widetilde{\mathcal{E}}_{h}\right)=O(\epsilon)
$$

and as $\epsilon>0$ was arbitrary, we obtain the result. 
Remark 4.3. Note that the proof gives no information about the exact vanishing rate of $D\left(\widetilde{\mathcal{E}}_{h}\right)$ as $h \rightarrow 0$. For this, one must have information on the dependence of $C(\epsilon)$ on $\epsilon$, and then optimize in $\epsilon$ in 4.8 as $h \rightarrow 0$. This is what we do in Section 6 to obtain improved remainders in the case of scattering by the disk.

To prove Lemma 4.1, we use theorems from [14. The following theorem follows from [14, ch. 2, eq. 2.42]:

Theorem 4.4 (Erdös-Turán). There is a constant $c>0$ such that if

$$
\omega=\left\{e^{2 \pi i x_{1}}, \ldots, e^{2 \pi i x_{N}}\right\}
$$

is a finite sequence of $N$ points on $\mathbb{S}^{1}$ and $m$ is any positive integer, then

$$
D(\omega) \leq c\left(\frac{1}{m}+\sum_{j=1}^{m} \frac{1}{j}\left|\frac{1}{N} \sum_{l=1}^{N} e^{2 \pi i j x_{l}}\right|\right) .
$$

To bound the exponential sums that appear on the right hand side of 4.9 , we use 14 , ch. 1, thm. 2.7], namely

Theorem 4.5. Let $a$ and $b$ be integers with $a<b$, and let $f$ be twice differentiable on $[a, b]$ with $\left|f^{\prime \prime}(x)\right| \geq \rho>0$ for $x \in[a, b]$. Then

$$
\left|\sum_{l=a}^{b} e^{2 \pi i f(l)}\right| \leq\left(\left|f^{\prime}(b)-f^{\prime}(a)\right|+2\right)\left(\frac{4}{\sqrt{\rho}}+3\right) .
$$

We also need [14, thm. 2.6] (with minor modifications in notation):

Theorem 4.6. For $1 \leq i \leq k$, let $\omega_{i}$ be a set of $\left|\omega_{i}\right|$ points on $\mathbb{S}^{1}$ with discrepancy $D\left(\omega_{i}\right)$. Let $\omega$ be a concatenation of $\omega_{1}, \ldots, \omega_{k}$, that is, a set obtained by listing in some order the terms of the $\omega_{i}$. Then

$$
D(\omega) \leq \sum_{i=1}^{k} \frac{\left|\omega_{i}\right|}{|\omega|} D\left(\omega_{i}\right),
$$

where $|\omega|$ is the number of points in $\omega$.

Proof of Lemma 4.1. We begin by assuming that $G^{\prime \prime}$ has no zeroes in the open interval $(0, R)$.

We first analyze the subset $\mathcal{E}_{h}(\epsilon) \subset \mathcal{E}_{h}$ defined in 4.6 . Define

$$
\begin{aligned}
& \tilde{\rho}=\tilde{\rho}(\epsilon)=\min _{\epsilon \leq \alpha \leq R-\epsilon}\left|G^{\prime \prime}(\alpha)\right| \\
& \tilde{\kappa}=\tilde{\kappa}(\epsilon)=2 \max _{\epsilon \leq \alpha \leq R-\epsilon}\left|G^{\prime}(\alpha)\right| .
\end{aligned}
$$

We will show that for each $\gamma \in(0,1)$ there is a constant $c=c(\gamma)>0$ so that for each $\epsilon>0$,

$$
D\left(\mathcal{E}_{h}(\epsilon)\right)<c\left(h^{\gamma}+\tilde{\kappa} \tilde{\rho}^{-1 / 2} h^{1 / 2-\gamma / 2}+\tilde{\rho}^{-1 / 2} h^{1 / 2}+\tilde{\kappa} h^{1-\gamma}\right) .
$$

Since $\mathcal{E}_{h}(\epsilon)-\mathcal{E}_{h}=h^{-d+1}(O(\epsilon)+O(h))$, for some $c=c(\gamma)>0$ independent of $\epsilon$ we have

$$
\begin{aligned}
D\left(\mathcal{E}_{h}\right) & =\sup _{0 \leq \phi_{0}<\phi_{1} \leq 2 \pi}\left|\frac{N\left(\phi_{0}, \phi_{1} ; \mathcal{E}_{h}\right)}{\left|\mathcal{E}_{h}\right|}-\frac{\phi_{1}-\phi_{0}}{2 \pi}\right| \\
& \leq c(\epsilon+h)+D\left(\mathcal{E}_{h}(\epsilon)\right),
\end{aligned}
$$


showing that

$$
\limsup _{h \rightarrow 0} D\left(\mathcal{E}_{h}\right) \leq c \epsilon
$$

Since $\epsilon>0$ is arbitrary, this gives 4.4). Thus it remains to prove (4.13).

Case 1: dimension $d=2$. Note that when $d=2$ the multiplicity of the eigenspaces is $p_{2}(l)=1$ if $l=0$ and 2 otherwise, so that

$$
\left|\mathcal{E}_{h}(\epsilon)\right|=2(\lfloor(R-\epsilon) / h\rfloor-\lceil\epsilon / h\rceil+1) .
$$

We apply Theorem 4.4 with $\omega=\mathcal{E}_{h}(\epsilon)$, so that, in the notation of Theorem $4.4, x_{l}=$ $G(l h) /(2 \pi h)$. Thus

$$
D\left(\mathcal{E}_{h}(\epsilon)\right) \leq c\left(\frac{1}{m}+\sum_{j=1}^{m} \frac{1}{j}\left|\frac{1}{\lfloor(R-\epsilon) / h\rfloor-\lceil\epsilon / h\rceil+1} \sum_{l=\lceil\epsilon / h\rceil}^{\lfloor(R-\epsilon) / h\rfloor} e^{i j G(l h) / h}\right|\right)
$$

Then we apply Theorem 4.5 with $f(x)=(j / 2 \pi) G(x h) / h, a=\lceil\epsilon / h\rceil$, and $b=\lfloor(R-\epsilon) / h\rfloor$. Thus, if $x h \leq R-\epsilon$ then $\left|f^{\prime \prime}(x)\right|=h j\left|G^{\prime \prime}(x h)\right| / 2 \pi \geq h j \tilde{\rho} / 2 \pi$, which equals $\rho$ in the notation of Theorem 4.5. It follows that

$$
D\left(\mathcal{E}_{h}(\epsilon)\right) \leq c\left(\frac{1}{m}+\frac{h}{R-2 \epsilon-h} \sum_{j=1}^{m} \frac{1}{j}\left(\frac{j \tilde{\kappa}}{\pi}+2\right)\left(\left(\frac{32 \pi}{h j \tilde{\rho}}\right)^{1 / 2}+3\right)\right) .
$$

By letting $m=\left\lfloor h^{-\gamma}\right\rfloor$ for any $\gamma>0$, we obtain (4.13).

Finally, suppose there are a finite number of points $0<a_{1}<\ldots<a_{n-1}<R$ with $G^{\prime \prime}\left(\alpha_{i}\right)=0$, and let $a_{0}=0, a_{n}=R$. Note that, if we define $\mathcal{E}_{h}(a, b)$ to be the set of $x_{l, k}$ with $a \leq l h \leq b$, counted with multiplicity, then the above arguments show that $\lim _{h \rightarrow 0} D\left(\mathcal{E}_{h}(a, b)\right)=0$; in fact if $\widetilde{\rho}(\epsilon, h)$ (resp. $\widetilde{\kappa}$ ) is defined to be $\min _{a+\epsilon \leq \alpha \leq b-\epsilon}\left|G^{\prime \prime}(\alpha)\right|$ (resp. $\max \left|G^{\prime}(\alpha)\right|$ ), then the proof is the same. The lemma in the $d=2$ case now follows from Theorem 4.6 since by 4.11

$$
D\left(\mathcal{E}_{h}\right) \leq \sum_{i=1}^{n} D\left(\mathcal{E}_{h}\left(a_{i-1}, a_{i}\right)\right)
$$

The proof is now complete in the case $d=2$.

Case 2: dimension $d>2$. As in the $d=2$ case, we begin by assuming that $G^{\prime \prime}(\alpha)$ has zeroes only at 0 and $R$. We now have to deal with the increasing multiplicities $p_{d}(l)$.

We will apply Theorem 4.6 to $D\left(\mathcal{E}_{h}(\epsilon)\right)$ decomposed as a superposition in the following way. It will be convenient to set

$$
N:=\lfloor(R-\epsilon) / h\rfloor .
$$

Define,

$$
\left.\omega(n):=\left\{e^{i G(l h) / h}: n \leq l \leq N\right\rfloor\right\}
$$


with unit multiplicity. Note that $\omega(n)$ has $N-n+1$ elements. Setting

$$
\begin{aligned}
\omega_{1} & =\omega(0) \\
\omega_{2} & =\cdots=\omega_{p_{d}(1)}=\omega(1) \\
\omega_{p_{d}(1)+1} & =\cdots=\omega_{p_{d}(2)}=\omega(2) \\
\vdots & \\
\omega_{p_{d}(N-1)+1} & =\cdots=\omega_{p_{d}(N)}=\omega(N) .
\end{aligned}
$$

we see that the set $\mathcal{E}_{h}(\epsilon)$ is the superposition of the sets $\omega_{1}, \ldots \omega_{p_{d}(N)}$.

The discrepancy $D(\omega(n))$ can be estimated using the method from the $d=2$ case. In particular, as in 4.15 we see that for any positive integer $m$,

$$
D(\omega(n)) \leq c\left(\frac{1}{m}+\sum_{j=1}^{m} \frac{1}{(N-n+1) j}\left|(\tilde{\kappa} j+2)\left(\left(\frac{32}{j c \tilde{\rho} h}\right)^{1 / 2}+3\right)\right|\right)
$$

By Theorem 4.6, we have

$$
\begin{aligned}
D\left(\mathcal{E}_{h}(\epsilon)\right) & \leq \sum_{i=0}^{p_{d}(N)} \frac{\left|\omega_{i}\right|}{\left|\mathcal{E}_{h}(\epsilon)\right|} D\left(\omega_{i}\right) \\
& \leq \frac{1}{\left|\mathcal{E}_{h}(\epsilon)\right|} \sum_{n=0}^{N}|\omega(n)|\left(p_{d}(n)-p_{d}(n-1)\right) D(\omega(n)) \\
& \leq \operatorname{ch}^{d-1} \sum_{n=1}^{N}(N-n+1)(n+1)^{d-3} D(\omega(n)),
\end{aligned}
$$

Substituting the estimate (4.17) into (4.18), again with $m=\left\lfloor h^{-\gamma}\right\rfloor$ for some fixed $\gamma \in(0,1)$, we end up with five terms to deal with corresponding to the five terms in the right hand side of (4.17). For all of these we use standard bounds for sums of polynomials and $N \sim c / h$. The easiest is the $1 / m$ term, since

$$
h^{d-1} \sum_{n=1}^{N}(N-n+1)(n+1)^{d-3} h^{\gamma} \leq c h^{\gamma} .
$$

Next we do the terms involving $\tilde{\rho}$. There is

$$
\begin{aligned}
& h^{d-1} \sum_{n=1}^{N}(N-n+1)(n+1)^{d-3} \sum_{j=1}^{\left\lfloor h^{-\gamma}\right\rfloor} \frac{1}{(N-n+1) j}(\tilde{\kappa} j)\left(\frac{32}{j c \tilde{\rho} h}\right)^{1 / 2} \\
& \leq c h^{d-1} \tilde{\kappa} \sum_{n=1}^{N}(n+1)^{d-3} \sum_{j=1}^{\left\lfloor h^{-\gamma}\right\rfloor}\left(\frac{32}{j \tilde{\rho} h}\right)^{1 / 2} \\
& \leq c h^{d-1} \tilde{\kappa}\left(\frac{1}{\tilde{\rho} h}\right)^{1 / 2} \sum_{n=1}^{N}(n+1)^{d-3} \sum_{j=1}^{\left\lfloor h^{-\gamma}\right\rfloor} j^{-1 / 2} \\
& \leq c\left(\frac{1}{\tilde{\rho}}\right)^{1 / 2} \tilde{\kappa} h^{1 / 2-\gamma / 2}
\end{aligned}
$$


and

$$
\begin{aligned}
& h^{d-1} \sum_{n=1}^{N}(N-n+1)(n+1)^{d-3} \sum_{j=1}^{\left\lfloor h^{-\gamma}\right\rfloor} \frac{2}{(N-n+1) j}\left(\frac{32}{j c \tilde{\rho} h}\right)^{1 / 2} \\
& \leq h^{d-1} c\left(\frac{1}{\tilde{\rho} h}\right)^{1 / 2} \sum_{n=1}^{N}(n+1)^{d-3} \sum_{j=1}^{\left\lfloor h^{-\gamma}\right\rfloor} j^{-3 / 2} \\
& \leq c\left(\frac{1}{\tilde{\rho}}\right)^{1 / 2} h^{-1 / 2} .
\end{aligned}
$$

The other terms are

$$
\begin{aligned}
& h^{d-1} \sum_{n=1}^{N}(N-n+1)(n+1)^{d-3} \sum_{j=1}^{\left\lfloor h^{-\gamma}\right\rfloor} \frac{1}{(N-n+1) j}(\tilde{\kappa} j) \times 3 \\
& \leq c \tilde{\kappa} h^{1-\gamma} \leq c \tilde{\kappa} h^{1 / 2-\gamma / 2},
\end{aligned}
$$

and

$$
h^{d-1} \sum_{n=1}^{N}(N-n+1)(n+1)^{d-3} \sum_{j=1}^{\left\lfloor h^{-\gamma}\right\rfloor} \frac{6}{(N-n+1) j} \leq \operatorname{chlog}(1 / h) .
$$

Combining (4.19) - 4.23) with (4.18) gives 4.13).

We take care of the case of a non-trivial number of zeroes of $G^{\prime \prime}$ on $[0, R]$ exactly as in the $d=2$ case. This completes the proof of Lemma 4.1

We can now prove Theorem 1.2 .

Proof of Theorem 1.2. By Theorem 1.1, the eigenvalues of the scattering matrix for $0 \leq l \leq$ $R / h$ are given by

$$
\exp \left\{\frac{i}{h}\left(G\left(\left(l+\frac{d-2}{2}\right) h\right)\right)\right\}+O(h)
$$

in the case of even dimension $d$, and the same in odd dimensions away from any $\epsilon$ neighbourhood of the set $S=\left\{\alpha \mid G^{\prime}(\alpha) / \pi \in \mathbb{Z}\right\}$, where $\alpha=l / h$. Since by assumption $\Sigma=G^{\prime}$ satisfies (1.9), $G$ satisfies 4.2 , so the conclusion of Lemma 4.1 holds for $G$. Finally, 4.2 . implies that $S$ is a finite set, so we can apply Proposition 4.2 proving $(1.13)$ and hence Theorem 1.2

\section{Examples of potentials that satisfy Assumption 1.9}

We use expression (2.6) for the scattering angle to prove

Proposition 5.1. Suppose that on the region of interaction $\mathcal{R}$ the potential $V$ satisfies

$$
V^{\prime}(r) \leq 0 \text { and }\left(V^{\prime}\right)^{2}+(1-V)\left(V^{\prime}+r V^{\prime \prime}\right)>0 \text { for } r<R .
$$

Then $\Sigma^{\prime}(\alpha)<0$ for for $\alpha \in[0, R)$. 
The conditions in (5.1) hold in particular for $V=c W$, where $c$ is sufficiently large and where $W(r)=0$ for $r \geq R, W(r)>0$ for $0 \leq r<R$ and $W^{\prime \prime}(r)$ is positive and monotone decreasing in some nonempty interval $[R-\epsilon, R)$. An explicit example is

$$
W(r)= \begin{cases}e^{1 /\left(r^{2}-R^{2}\right)}, & r<R \\ 0, & r \geq R .\end{cases}
$$

Proof. In (2.6), set $s=r / r_{m}$, so

$$
\begin{aligned}
\Sigma(\alpha) & =\pi-2 \int_{r_{m}}^{\infty} \frac{\alpha}{\left(s r_{m}\right)^{2} \sqrt{1-\alpha^{2} /\left(\left(s r_{m}\right)^{2}-V\left(s r_{m}\right)\right.}} d\left(s r_{m}\right) \\
& =\pi-2 \int_{1}^{\infty} \frac{\alpha}{s^{2} \sqrt{r_{m}^{2}-\alpha^{2} / s^{2}-r_{m}^{2} V\left(s r_{m}\right)}} d s .
\end{aligned}
$$

Differentiating under the integral sign gives

$$
\begin{aligned}
-\frac{1}{2} \Sigma^{\prime}(\alpha)= & \int_{1}^{\infty}\left(\frac{1}{s^{2} \sqrt{r_{m}^{2}-\alpha^{2} / s^{2}-r_{m}^{2} V\left(s r_{m}\right)}}\right) \\
& \left.-\frac{\alpha}{2} \frac{2 r_{m} r_{m}^{\prime}-2 \alpha / s^{2}-2 r_{m} V\left(s r_{m}\right) r_{m}^{\prime}-r_{m}^{2} s V^{\prime}\left(s r_{m}\right) r_{m}^{\prime}}{s^{2}\left(r_{m}^{2}-\alpha^{2} / s^{2}-r_{m}^{2} V\left(s r_{m}\right)\right)^{3 / 2}}\right) d s \\
= & \int_{1}^{\infty} \frac{\left(r_{m}^{2}\left(1-V\left(s r_{m}\right)\right)-\alpha r_{m} r_{m}^{\prime}\left(1-V\left(s r_{m}\right)-\frac{1}{2} r_{m} s V^{\prime}\left(s r_{m}\right)\right)\right.}{s^{2}\left(r_{m}^{2}-\alpha^{2} / s^{2}-r_{m}^{2} V\left(s r_{m}\right)\right)^{3 / 2}} d s
\end{aligned}
$$

Differentiating

$$
1-\alpha^{2} / r_{m}^{2}-V\left(r_{m}\right)=0
$$

shows $\alpha r_{m} r_{m}^{\prime}=\alpha^{2}\left(1-V\left(r_{m}\right)-\frac{1}{2} r_{m} V^{\prime}\left(r_{m}\right)\right)^{-1}$. Plugging this in gives

$$
\begin{aligned}
-\frac{1}{2} \Sigma^{\prime}(\alpha)= & \int_{1}^{\infty}\left(r_{m}^{2}\left(1-V\left(s r_{m}\right)\right)-\alpha^{2} \frac{1-V\left(s r_{m}\right)-\frac{1}{2} r_{m} s V^{\prime}\left(s r_{m}\right)}{1-V\left(r_{m}\right)-\frac{1}{2} r_{m} V^{\prime}\left(r_{m}\right)}\right) \\
& \times \frac{1}{s^{2}\left(r_{m}^{2}-\alpha^{2} / s^{2}-r_{m}^{2} V\left(s r_{m}\right)\right)^{3 / 2}} d s
\end{aligned}
$$

and using (5.2) again shows that $(1 / 2) \Sigma^{\prime}(\alpha)$ is equal to

$$
\begin{aligned}
\int_{1}^{\infty} & \frac{1-V\left(s r_{m}\right)-\frac{1}{2} r_{m} s V^{\prime}\left(s r_{m}\right)}{s^{2}\left(r_{m}^{2}-\alpha^{2} / s^{2}-r_{m}^{2} V\left(s r_{m}\right)\right)^{3 / 2}} \\
& \times\left(\frac{1-V\left(s r_{m}\right)}{1-V\left(s r_{m}\right)-\frac{1}{2} r_{m} s V^{\prime}\left(s r_{m}\right)}-\frac{1-V\left(r_{m}\right)}{1-V\left(r_{m}\right)-\frac{1}{2} r_{m} V^{\prime}\left(r_{m}\right)}\right) r_{m}^{2} d s \\
= & \int_{r_{m}}^{\infty} \frac{1-V(r)-\frac{1}{2} r V^{\prime}(r)}{r^{2}\left(1-\alpha^{2} / r^{2}-V(r)\right)^{3 / 2}}\left(\frac{1-V(r)}{1-V(r)-\frac{1}{2} r V^{\prime}(r)}-\frac{1-V\left(r_{m}\right)}{1-V\left(r_{m}\right)-\frac{1}{2} r_{m} V^{\prime}\left(r_{m}\right)}\right) d r
\end{aligned}
$$

Differentiating the expression $(1-V(r)) /\left(1-V(r)-\frac{1}{2} r V^{\prime}(r)\right)$ with respect to $r$ and using $V^{\prime} \leq 0$, we see that the integrand is positive if for $r_{m}<r<R$ if

$$
r\left(V^{\prime}\right)^{2}+(1-V)\left(V^{\prime}+r V^{\prime \prime}\right)>0
$$

These conditions are implied by (5.1) in the first paragraph of the proposition.

To check that the condition in the second paragraph is sufficient, observe the following. If $V(r) \geq 0, V^{\prime}(r)<0$ and $V^{\prime \prime}(r)>0$ on some open interval $(R-\epsilon, R)$, it follows that for 
$r$ sufficiently close to $R$, that $V^{\prime}+r V^{\prime \prime}>0$. By picking large enough $c>0$, 5.3 will hold on the region of interaction $\mathcal{R}$.

Remark 5.2. One can ask whether there exist potentials for which equidistribution fails. It is clear from Theorem 1.1 that the scattering matrix for $V$ will fail to be equidistributed if the scattering angle $\Sigma(\alpha)$ associated to $V$ is equal to a constant rational multiple of $2 \pi$ on some interval with $\alpha<R$. So we can ask whether there exists such a potential. Let $\mathcal{S}$ be the map (2.6) taking $V$ to its scattering angle $\Sigma$. Linearizing $\mathcal{S}$ at the zero potential gives an integral operator which is an elliptic pseudodifferential operator of order $1 / 2$ (apart from an extra singularity at $r=\alpha=0$ ). This makes it seem likely to the authors that the range of $\mathcal{S}$ is quite large, very likely including scattering angles such as described above that would imply non-equidistribution.

\section{Scattering By The Disk}

In this section we will prove Theorem 1.8 from the introduction. We restrict our attention to the ball of radius 1 , since the phase shifts for the ball of radius $R$ can be obtained from those for $R=1$ by a scaling argument.

Here we use an ODE analogous to that in (1.3) to give a formula for the eigenvalues. In fact, for any smooth solution $f_{l}$ to $\Delta_{\mathbb{S}^{d-1}} f_{l}=l(l+d-2) f_{l}$, a straightforward computation shows that

$$
S_{k}\left(f_{l}\right)=-\frac{H_{l+(d-2) / 2}^{(1)}(k)}{H_{l+(d-2) / 2}^{(2)}(k)} f_{l}
$$

where the $H_{\nu}^{(i)}$ are Hankel functions of order $\nu$ [1]. It follows that

$$
S_{k}\left(f_{l}\right)=e^{i x_{k, l}} f_{l}, \quad x_{k, l}=2 \arg H_{l+(d-2) / 2}^{(1)}(k)+\pi .
$$

We now prove the first part of Theorem 1.8, which amounts to determining the asymptotics of the argument of the Hankel function when $l / k \leq 1-k^{-1 / 3}$. Let us define in this section $\nu=l+(d-2) / 2$ and $\alpha=\nu / k$, and study the range $\alpha \leq 1-k^{-1 / 3}$.

We first consider the range where $\alpha$ is small, say $\alpha \leq 3 / 4$. Then we use the expressions [1, 9.1.22] for $J_{\nu}$ and $Y_{\nu}$ to derive

$$
H_{\alpha k}^{(1)}(k)=\frac{1}{\pi} \int_{0}^{\pi} e^{i k(\sin \theta-\alpha \theta)} d \theta-(\text { integrals from } 0 \text { to } \infty) .
$$

It is easy to bound the integrals from 0 to $\infty$ by $O\left(k^{-1}\right)$ uniformly for $\alpha \leq 3 / 4$. On the other hand, stationary phase applied to the $\theta$ integral gives

$$
H_{\alpha k}^{(1)}(k)=\sqrt{\frac{2}{\pi k}}\left(1-\alpha^{2}\right)^{-1 / 4} e^{i k\left(\sqrt{1-\alpha^{2}}-\alpha \cos ^{-1} \alpha\right)} e^{-i \pi / 4}+O\left(k^{-1}\right),
$$

from which it follows that

$$
2 \arg H_{\alpha k}^{(1)}(k)=2 k\left(\sqrt{1-\alpha^{2}}-\alpha \cos ^{-1} \alpha\right)-\pi / 2+O\left(k^{-1 / 2}\right)
$$

in this range. In view of 6.2 and 1.17 this proves 1.16 in the range $\alpha \leq 3 / 4$.

In the range $1 / 2 \leq \alpha \leq 1-k^{-1 / 3}$, we use the asymptotic formulae [1, 9.3.35, 9.3.36] which shows that

$$
H_{\alpha k}^{(1)}(k)=\left(\frac{-4 \zeta}{\alpha^{-2}-1}\right)^{1 / 4} \nu^{-1 / 3}\left((\mathrm{Ai}-i \mathrm{Bi})\left(\nu^{2 / 3} \zeta\right)\right)\left(1+O\left(k^{-2}\right)\right)+O\left(k^{-3 / 2}\right), \quad \nu=\alpha k
$$


where $\zeta=\zeta(\alpha)$ is defined by

$$
\frac{2}{3}(-\zeta)^{3 / 2}=\int_{1}^{\alpha^{-1}} \frac{\sqrt{t^{2}-1}}{t} d t=\sqrt{\alpha^{-2}-1}-\cos ^{-1}(\alpha) ;
$$

notice that $\zeta$ is real and negative for $\alpha<1$, and $-\zeta \sim c(1-\alpha)$ for some positive $c$ as $\alpha \rightarrow 1$. To derive (6.4) from [1, 9.3.35, 9.3.36] we used the fact that $\zeta$ lies in a compact set in this range of $\alpha$, that the $a_{k}$ and $b_{k}$ are therefore uniformly bounded, that $\nu$ and $k$ are comparable when $\alpha \in[1 / 2,1]$ and finally that we have bounds

$$
\left|\operatorname{Ai}^{\prime}\left(\nu^{2 / 3} \zeta\right)\right|+\left|\operatorname{Bi}^{\prime}\left(\nu^{2 / 3} \zeta\right)\right| \leq C \nu^{1 / 6}
$$

uniformly for $\zeta$ in this range - see [1, 10.4.62, 10.4.67]. It follows that

$$
2 \arg H_{\alpha k}^{(1)}(k)=2\left((\mathrm{Ai}-i \mathrm{Bi})\left(\alpha^{2 / 3} k^{2 / 3} \zeta\right)\right)+O\left(k^{-7 / 6}\right) .
$$

Finally using the asymptotics [1, 10.4.60, 10.4.64], we get

$$
(\mathrm{Ai}-i \mathrm{Bi})\left(\alpha^{2 / 3} k^{2 / 3} \zeta\right)=\frac{\left(\alpha k(-\zeta)^{3 / 2}\right)^{-1 / 6}}{\pi^{1 / 2}}\left((-i) e^{i\left(\frac{2}{3} \alpha k(-\zeta)^{3 / 2}+\pi / 4\right)}+O\left(k^{-1}(-\zeta)^{-3 / 2}\right)\right)
$$

It follows, using the explicit expression for $\zeta(\alpha)$ in 6.5 , that

$$
2 \arg H_{\alpha k}^{(1)}(k)=2 k\left(\sqrt{1-\alpha^{2}}-\alpha \cos ^{-1}(\alpha)\right)-\pi / 2+O\left(k^{-1}(1-\alpha)^{-3 / 2}\right) .
$$

Since we have taken $1-\alpha \geq k^{-1 / 3}$, that gives us (1.16). (Pleasingly, we get the same expression as in (6.3), a useful check on the computations.)

We now turn to the proof of equidistribution. We first note that, as in the proof of Proposition 4.2 (with $S=\{1\}$ and $\epsilon=k^{-1 / 3}$ ) the discrepancy of the exact eigenvalues $e^{i x_{k, l}}$ is equal to that of the approximate eigenvalues $e^{i k G_{b}(\alpha)-\pi / 2}$ up to an error $O\left(k^{-1 / 3}\right)$ which is acceptable. So it suffices to prove (1.18) for the approximations $e^{i k G_{b}(\alpha)-\pi / 2}$. We apply (4.13), using

$$
\begin{aligned}
& \widetilde{\kappa} \leq \max _{0 \leq \alpha \leq 1}\left|G^{\prime}\right| \leq \pi, \\
& \widetilde{\rho} \geq \min _{0 \leq \alpha \leq 1} G^{\prime \prime}(\alpha) \geq c>0 .
\end{aligned}
$$

This means that in 4.13, $\tilde{\rho}(\epsilon, 1 / k) \geq c>0$ and $\tilde{\kappa}(\epsilon, 1 / k) \leq \pi$ for all $\epsilon$, and thus for any $0<\gamma<1$,

$$
D\left(\mathcal{E}_{1 / k}(\epsilon)\right) \leq C\left(k^{-\gamma}+k^{-1 / 2+\gamma / 2}\right) .
$$

Choosing $\gamma=1 / 3$ completes the proof.

\section{REFERENCES}

[1] M. Abramowitz and I. A. Stegun. Handbook of mathematical functions with formulas, graphs, and mathematical tables, volume 55 of National Bureau of Standards Applied Mathematics Series. For sale by the Superintendent of Documents, U.S. Government Printing Office, Washington, D.C., 1964.

[2] I. Alexandrova. Structure of the semi-classical amplitude for general scattering relations. Comm. Partial Differential Equations, 30(10-12):1505-1535, 2005.

[3] M. S. Birman and D. R. Yafaev. Asymptotics of the spectrum of the s-matrix in potential scattering. Soviet Phys. Dokl., 25(12):989-990, 1980.

[4] M. S. Birman and D. R. Yafaev. Asymptotic behavior of limit phases for scattering by potentials without spherical symmetry. Theoret. Math. Phys., 51(1):344-350, 1982.

[5] M. S. Birman and D. R. Yafaev. Asymptotic behaviour of the spectrum of the scattering matrix. $J$. Sov. Math., 25:793-814, 1984. 
[6] M. S. Birman and D. R. Yafaev. Spectral properties of the scattering matrix. St Petersburg Math. Journal, 4(6):1055-1079, 1993.

[7] D. Bulger and A. Pushnitski. The spectral density of the scattering matrix for high energies. arXiv:1110.3710, 2011.

[8] E. Doron and U. Smilansky. Semiclassical quantization of chaotic billiards: a scattering theory approach. Nonlinearity, 5(5):1055-1084, 1992.

[9] V. Guillemin. Sojourn times and asymptotic properties of the scattering matrix. In Proceedings of the Oji Seminar on Algebraic Analysis and the RIMS Symposium on Algebraic Analysis (Kyoto Univ., Kyoto, 1976), volume 12, pages 69-88, 1976/77 supplement.

[10] A. Hassell and J. Wunsch. The semiclassical resolvent and the propagator for non-trapping scattering metrics. Adv. Math., 217(2):586-682, 2008.

[11] L. Hörmander. Fourier integral operators. I. Acta Math., 127(1-2):79-183, 1971.

[12] L. Hörmander. The analysis of linear partial differential operators. I. Classics in Mathematics. SpringerVerlag, Berlin, 1983.

[13] L. Hörmander. The analysis of linear partial differential operators. III. Classics in Mathematics. Springer-Verlag, Berlin, 2007. Pseudo-differential Operators, Reprint of the 1994 edition.

[14] L. Kuipers and H. Niederreiter. Uniform distribution of sequences. Wiley-Interscience [John Wiley \& Sons], New York, 1974. Pure and Applied Mathematics.

[15] L. D. Landau and E. M. Lifshitz. Quantum mechanics: nonrelativistic theory. Pergamon Press, Oxford, 1965. Second revised edition.

[16] A. Majda. High frequency asymptotics for the scattering matrix and the inverse problem of acoustical scattering. Comm. Pure Appl. Math., 29(3):261-291, 1976.

[17] R. Melrose. Geometric Scattering Theory. Cambridge University Press, Cambridge, 1995.

[18] R. Melrose and M. Zworski. Scattering metrics and geodesic flow at infinity. Invent. Math., 124(13):389-436, 1996.

[19] R. Newton. Scattering Theory of Waves and Particles. McGraw-Hill, New York, 1965.

[20] V. Petkov and M. Zworski. Semi-classical estimates on the scattering determinant. Ann. Henri Poincaré, 2(4):675-711, 2001.

[21] M. Reed and B. Simon. Methods of modern mathematical physics. III. Academic Press [Harcourt Brace Jovanovich Publishers], New York, 1979.

[22] D. Robert and H. Tamura. Asymptotic behavior of scattering amplitudes in semi-classical and low energy limits. Ann. Inst. Fourier (Grenoble), 39(1):155-192, 1989.

[23] J. Sjöstrand and M. Zworski. Complex scaling and the distribution of scattering poles. J. Amer. Math. Soc., 4(4):729-769, 1991.

[24] A. V. Sobolev and D. R. Yafaev. Phase analysis in the problem of scattering by a radial potential. Zap. Nauchn. Sem. Leningrad. Otdel. Mat. Inst. Steklov. (LOMI), 147:155-178, 206, 1985. Boundary value problems of mathematical physics and related problems in the theory of functions, No. 17.

[25] D. Yafaev. Mathematical Scattering Theory: analytic theory. American Mathematical Society, Providence, RI, 2010.

[26] D. R. Yafaev. On the asymptotics of scattering phases for the Schrödinger equation. Ann. Inst. H. Poincaré Phys. Théor., 53(3):283-299, 1990.

[27] S. Zelditch. Index and dynamics of quantized contact transformations. Ann. Inst. Fourier (Grenoble), 47(1):305-363, 1997.

[28] S. Zelditch and M. Zworski. Spacing between phase shifts in a simple scattering problem. Comm. Math. Phys., 204(3):709-729, 1999. 
Department of Mathematics, Massachusettes Institute of Technology

E-mail address: datchev@math.mit.edu

Mathematical Sciences Institute, Australian National University and Department of MathEMATICS, UNIVERSITY OF TORONTO

E-mail address: jgell@math.toronto.edu

Mathematical Sciences Institute, Australian National University

E-mail address: Andrew.Hassell@anu.edu.au

Mathematical Sciences Institute, Australian National University and Department of Mathematics, Princeton University

E-mail address: peterch@math.princeton.edu 